\title{
Rhodium- and Palladium-Catalyzed 1,5-Substitution Reactions of 2-En-4-yne Acetates and Carbonates with Organoboronic Acids
}

\author{
Muhammed Ücüncü, ${ }^{+}$Erman Karakus, ${ }^{+}$Melih Kus, ${ }^{+}$Gürkan Eray Akpınar, ${ }^{+}$Özge Aksın-Artok, ${ }^{\dagger}$ \\ Norbert Krause, ${ }^{\dagger}$ Sila Karaca, ${ }^{+}$Nuran Elmaci, ${ }^{\dagger}$ and Levent Artok ${ }^{*,+}$ \\ ${ }^{\dagger}$ Department of Chemistry, Faculty of Science, Izmir Institute of Technology, Urla 35430, Izmir, Turkey \\ ${ }^{\ddagger}$ Organic Chemistry II, Dortmund University of Technology, Otto-Hahn-Strasse 6, D-44227 Dortmund, Germany \\ S Supporting Information
}

ABSTRACT: Two methods involving the rhodium-catalyzed reaction of 2-en-4-yne acetates and the palladium-catalyzed reaction of 2-en-4-yne carbonates with organoboronic acids were investigated; both afforded exclusively the $(E)$-configured vinylallenes. The coordinative interaction of the rhodium with the acetate group promoted the $\delta$-elimination of $\mathrm{Rh}(\mathrm{I})$-OAc from the alkenylrhodium intermediate II in both syn and anti modes, with the syn-elimination being the major path. DFT calculations revealed that a conformer of this intermediate (II), which can lead to the $(E)$-configured vinylallene product via the syn-elimination mode, is energetically the most favorable conformer. The rhodium-catalyzed procedure is not applicable to reactions involving $(E)$-configured enyne acetates, because the geometry of the alkenylrhodium intermediate that is derived from the corresponding (E)-enyne acetate would not allow such coordinative interaction to occur. The palladium-catalyzed method, which proceeds through formation of the $\sigma$-vinylallenylpalladium intermediate, $\mathbf{B}$, is suitable for both the $(E)$ - and $(Z)$-configured enyne carbonates and appears to have a wider scope for both organoboronic acids and enyne substrates. The palladium-catalyzed reaction of an enantiomerically enriched enyne carbonate proceeded with racemization.

\section{INTRODUCTION}

Grignard, organocopper, organolithium, and organozinc reagents are traditional nucleophilic organometallic reagents that are used for carbon-carbon coupling reactions. However, these reagents are highly moisture- and air-sensitive, have very short shelflives, and can tolerate only few electrophilic groups. Hence, use of these organometallic reagents requires protection of electrophilic functional groups, strict anhydrous conditions, and an inert atmosphere.

For these reasons, there has been an increasing trend toward adopting reagents that are relatively cheap, easy-to-handle, and tolerant to a wide range of functionalities and solvents during $\mathrm{C}-\mathrm{C}$ bond-forming reactions. Importantly, the rather lower reactivity of these reagents is compensated by performing the reactions in the presence of transition metal catalysts.

A classical example of these efforts is transition-metalcatalyzed biaryl-forming reactions. Kumada, Negishi, Stille, and Suzuki couplings are well-known transition-metal-catalyzed reactions. All of them involve the coupling of aryl halides or pseudoaryl halides with different types of organometallic reagents. Among them, the Suzuki reaction is the most popular and has found wide application in industrial processes and in laboratory syntheses since it utilizes organoboron reagents that have several advantages over its competitors. ${ }^{1}$
Organoboron reagents, particularly those bearing aryl and alkenyl units, are convenient and versatile reagents because they are widely available, thermally stable, inert to water and oxygen, relatively nontoxic, and tolerant to a wide range of reactive functional groups. ${ }^{2}$ For these reasons, organoboron reagents are often used, with convenience, in transition-metal-catalyzed C-C bond-forming reactions. ${ }^{2,3}$

Some of the leading applications in the use of organoboron reagents as a source for soft nucleophiles are ${ }^{4}$ the rhodium(I)catalyzed conjugate addition of aryl- or alkenylboronic acids to $\alpha, \beta$-unsaturated reagents, ${ }^{4,5}$ addition of arylboronic acids to aldehydes via $\mathrm{Rh}(\mathrm{I})$ catalysis (which is an alternative to the commonly known Grignard reaction), ${ }^{6} \mathrm{Rh}(\mathrm{I})$-catalyzed hydroarylation of internal alkynes with aryl- or alkenylboronic reagents, ${ }^{7} 1,3-S_{N} 2^{\prime}$-type substitution reactions of propargylic reagents leading to allenic products, ${ }^{8}$ and $\mathrm{Rh}(\mathrm{I})$-catalyzed ringopening reactions of oxabicyclic alkenes with organoborons. ${ }^{9}$

It has been previously established that conjugated enynes with a leaving group at the allylic position undergo $1,5-\left(S_{N} 2^{\prime \prime}\right)$ substitution with organometallic nucleophiles leading to vinylallenes (Scheme 1).

Received: February 2, 2011

Published: June 12, 2011 
Scheme 1. Reaction of 2-En-4-yne Reagents and Organometals<smiles>[R]C=CC=C([R])C#[R]</smiles>

Scheme 2. Palladation of 2-En-4-yne Carbonate Leading to $\sigma$-Vinylallenylpalladium(II) Species

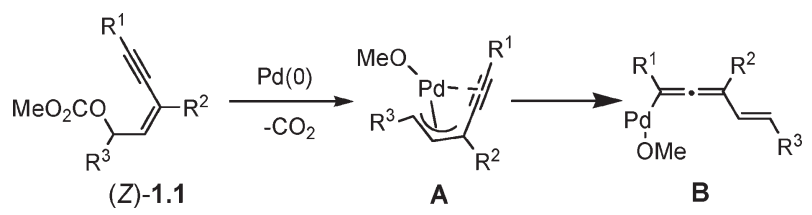

Goré and Dulcere found that the reaction of 2,4-enyne halides with methylmagnesium iodide or trimethylsilylmagnesium chloride (or trimethylsilyllithium) proceeds via an $S_{\mathrm{N}} 2^{\prime \prime}$-type nucleophilic substitution yielding an $E / Z$ mixture of vinylallenes. ${ }^{10}$ The method, however, is not general, since other Grignard reagents completely failed to form the desired vinylallenes. Krause et al. devised a more general method; they determined that $(E)$-2-en4-yne acetates can effectively undergo $S_{N} 2^{\prime \prime}$ type alkylation with various lithium cuprates affording an $E / Z$ mixture of vinylallenes. $^{11}$

However, to the best of our knowledge, there has been no report involving any 1,5-substitution reactions of 2,4-enynes with organoboron reagents, ${ }^{12}$ and therefore it was our intention to introduce the transition-metal-catalyzed organoboronic combination as a new method for the arylative and alkenylative reactions of 2-en-4-yne reagents. A part of our related study discloses that organoboron reagents can also be utilized as a nucleophilic aryl source for conjugated enyne acetates in the presence of a rhodium(I) complex to provide vinylallenes.

Another part of this report involves studies on the $\operatorname{Pd}(0)$ catalyzed reaction of organoboronic acids with conjugated enynes bearing a carbonate substituent in the allylic position. We have recently reported that the $\operatorname{Pd}(0)$-catalyzed carbonylation of carbonates of secondary $(Z)$-2-en-4-yne alcohols in the presence of $\mathrm{CO}$ in an alcohol medium proceeded through the formation of a $\sigma$-vinylallenylpalladium(II) complex (B) leading with high selectivity to 2,3,5-trienoates, while showing no traces of allylic substitution products (Scheme 2). ${ }^{13}$

It has been already shown that $\sigma$-allenylpalladium(II) species are also reactive toward transmetalation-type reactions with organoboronic acids leading to arylallenes. ${ }^{14}$ To this end, we purposely investigated the $\operatorname{Pd}(0)$-catalyzed 1,5-substitution reactivity of the 2,4-enyne carbonates with organoboronic reagents.

\section{RESULTS AND DISCUSSION}

Rh(I)-Catalyzed Reaction of 2-En-4-yne Acetates and Organoborons. The reaction of a $(Z)$-configured enyne acetate 1.1a with 3 equiv of phenylboronic acid proceeded in THF solvent, at room temperature, and in the presence of $[\mathrm{RhCl}(\operatorname{cod})]_{2}(6 \% \mathrm{Rh}), 3$ equiv of $\mathrm{KOH}$, and $0.1 \mathrm{~mL}$ of water to yield the desired phenyl-substituted vinylallene 3 aa product, which however was accompanied by significant amounts of
Table 1. Effect of Base and Water Additives on the $\mathrm{Rh}(\mathrm{I})$-Catalyzed Reaction of (Z)-Enyne Acetate 1.1a with Phenylboronic Acid

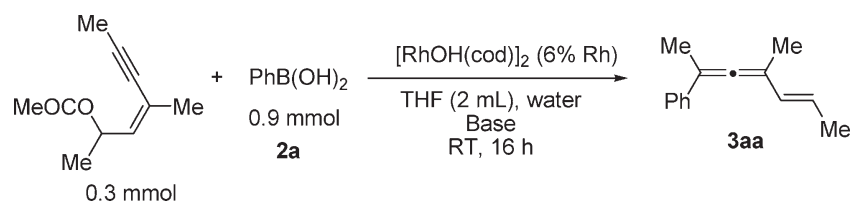

(Z)-1.1a

\begin{tabular}{clccc|} 
entry & base (equiv) & water $(\mathrm{mL})$ & conversion $(\%)^{a}$ & ${\text { yield, 3aa }(\%)^{a}}^{a}$ \\
$1^{b}$ & $\mathrm{KOH} \mathrm{(3)}$ & 0.1 & 78 & 58 \\
2 & $\mathrm{CsF}(3)$ & 0.1 & 100 & 55 \\
3 & $\mathrm{CsF}(2)$ & 0.1 & 100 & 75 \\
4 & $\mathrm{CsF}(1.5)$ & 0.1 & 100 & 85 \\
5 & $\mathrm{CsF}(1.1)$ & 0.1 & 95 & 75 \\
6 & $\mathrm{CsF}(1.5)$ & & 25 & 20 \\
7 & $\mathrm{CsF}(1.5)$ & 0.05 & 77 & 67 \\
8 & $\mathrm{CsF}(1.5)$ & 0.2 & 55 & 42 \\
9 & $\mathrm{KF}(3)$ & 0.1 & 40 & 38 \\
10 & $\mathrm{CsOH} \mathrm{(1.5)}_{11}$ & 0.1 & 100 & 50 \\
$\mathrm{Cs}_{2} \mathrm{CO}_{3}(1.5)$ & 0.1 & 82 & 40
\end{tabular}

${ }^{a}$ Determined by ${ }^{1} \mathrm{H}$ NMR using benzaldehyde as internal standard.

${ }^{b}$ Rhodium complex charged was $[\mathrm{RhCl}(\operatorname{cod})]_{2}$.

Table 2. Rh(I)-Catalyzed Reaction of the (Z)-Enyne Acetate 1.1a with Arylboronic Acids

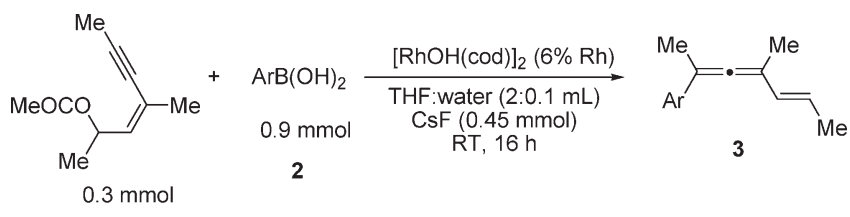

$(Z)-1.1 \mathrm{a}$

\begin{tabular}{|c|c|c|c|}
\hline entry & $\mathrm{Ar}$ & product & yield (\%) \\
\hline 1 & 2a Ph & $3 a a$ & 82 \\
\hline 2 & 2b $4-\mathrm{CF}_{3} \mathrm{C}_{6} \mathrm{H}_{4}$ & $3 a b$ & 76 \\
\hline 3 & 2c $4-\mathrm{MeCOC}_{6} \mathrm{H}_{4}$ & $3 a c$ & 77 \\
\hline 4 & 2d 4- $\mathrm{MeC}_{6} \mathrm{H}_{4}$ & 3ad & 83 \\
\hline 5 & 2e $3-\mathrm{MeC}_{6} \mathrm{H}_{4}$ & $3 a e$ & 86 \\
\hline 6 & $2 \mathrm{f} 3-\mathrm{ClC}_{6} \mathrm{H}_{4}$ & $3 \mathrm{af}$ & 77 \\
\hline 7 & $2 \mathrm{~g} 2-\mathrm{MeOC}_{6} \mathrm{H}_{4}$ & $3 a g$ & 83 \\
\hline 8 & 2h 2-naphthyl & $3 \mathrm{ah}$ & 76 \\
\hline 9 & 2i 3-thienyl & 3ai & 81 \\
\hline
\end{tabular}

nonseparable intricate isomers of the vinylallene with unassigned structures (Table 1, entry 1 ).

The reaction halted after a period of time, and the conversion was incomplete even with higher loadings of $\mathrm{Rh}$ at the beginning of the reaction, indicating that a change in the composition of the reaction medium (possibly due to an increased concentration of the acetate dispelled from the enyne substrate) lowered the activity of the rhodium significantly.

The complete conversion of $(Z)$-1.1a could be attained by substituting the base with CsF (entry 2) in the presence of $[\mathrm{RhOH}(\mathrm{cod})]_{2}$ complex. The formation of isomeric byproducts 
Table 3. $R h(I)$-Catalyzed Reaction of ( $Z$ )-Enyne Acetates with Phenylboronic Acid

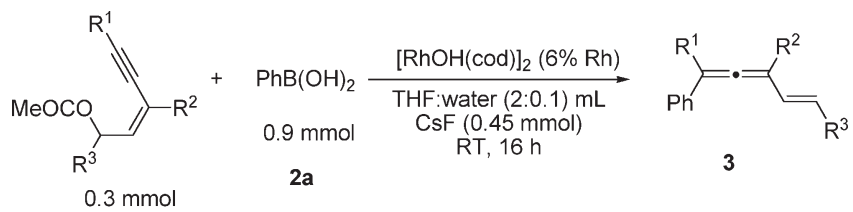

(Z)-1.1

\begin{tabular}{ccccccc} 
entry & substrate & $\mathrm{R}^{1}$ & $\mathrm{R}^{2}$ & $\mathrm{R}^{3}$ & product & isolated yield (\%) \\
1 & $(Z)-\mathbf{1 . 1 b}$ & $\mathrm{Bu}$ & $\mathrm{Me}$ & $\mathrm{Me}$ & 3ba & 72 \\
2 & $(Z)-\mathbf{1 . 1 c}$ & $\mathrm{Cy}$ & $\mathrm{Me}$ & $\mathrm{Me}$ & $3 \mathbf{c a}$ & 70 \\
3 & $(Z)-1.1 \mathrm{~d}$ & $\mathrm{Bu}$ & $\mathrm{Bu}$ & $\mathrm{Me}$ & $\mathbf{3 d a}$ & 75 \\
4 & $(Z)-\mathbf{1 . 1 e}$ & $\mathrm{Bu}$ & $\mathrm{Ph}$ & $\mathrm{Me}$ & $\mathbf{3 e a}$ & 73 \\
5 & $(Z)-\mathbf{1 . 1 f}$ & $\mathrm{Me}$ & $\mathrm{Me}$ & $\mathrm{Bu}$ & $\mathbf{3 f a}$ & 79 \\
6 & $(Z)-\mathbf{1 . 1 9}$ & $\mathrm{Me}$ & $\mathrm{Me}$ & $i-\mathrm{Pr}$ & $\mathbf{3 g a}$ & 77 \\
\hline
\end{tabular}

could be minimized and thereby high yields of 3aa product could be achieved by decreasing the $\mathrm{CsF}$ addition to 1.5 equiv (entries $2-5)$. The fine adjustment of the amount of added water seemed highly critical for the effectiveness of the process; the arylative substitution reaction did not proceed to completion when the reaction medium contained less or higher than $0.1 \mathrm{~mL}$ of water (entries 6-8). KF salt could not be an alternative flouride source (entry 9), and the use of other Cs compounds instead of CsF was useless (entries 10 and 11). ${ }^{15}$

The above-shown finding represents the first example of an arylative $S_{N} 2^{\prime \prime}$-type reaction for 2-en-4-yne reagents. In contrast to their transformation with organocopper and Grignard reagents, the resulting vinylallene had the vinylic group exclusively in $(E)$-configuration.

Having established the effective conditions, an array of substituted boronic acids were then subjected to the reactions with the enyne acetate $(Z)$-1.1a (Table 2 ). The reaction tolerated both electron-withdrawing and -donating groups on the meta and para positions, giving rise to high yields of the corresponding arylated vinylallene products $3 \mathrm{aa}-3 \mathrm{af}$ (entries $1-6)$. A sterically congested organoboronic acid (2g), 2-naphthylboronic acid, and 3-thienylboronic acid were also suitable components for the reaction, yielding related products $3 \mathrm{ag}-3 \mathrm{ai}$ with isolated yields in the range of $76-83 \%$ (entries $7-9$ ). ${ }^{16}$

The scope of the arylation method was also surveyed for a range of acetates of secondary $(Z)$-enynols. The method appeared suitable for the enyne acetates bearing butyl $((Z)-\mathbf{1 . 1 b})$ or cyclohexyl $((Z)-1.1 c)$ groups on the alkynyl moiety $\left(\mathrm{R}^{1}\right.$, Table 3, entries 1 and 2), butyl ((Z)-1.1d), or phenyl $((Z)-\mathbf{1 . 1 e})$ groups in the $\mathrm{R}^{2}$ position (entries 3 and 4$)$ and butyl $((Z)-\mathbf{1 . 1 f})$ or isopropyl $((Z)-\mathbf{1 . 1 g})$ groups on the allylic carbon $\left(\mathrm{R}^{3}\right.$, entries 5 and 6), affording the corresponding vinylallenes in good yields when reacted with phenylboronic acid $(70-79 \%){ }^{17}$

The $\mathrm{Rh}(\mathrm{I})$-catalyzed reaction of the acetate of an enantiomerically enriched enynol reagent, $(R, Z)-1.1 \mathrm{a}$ (94.5\% ee) with phenylboronic acid under the optimal conditions proceeded with partial racemization and yielded $(S)-(+)$-vinylallene as the major enantiomer (82\% isolated yield, $40 \%$ ee, $[\alpha]^{25}{ }_{\mathrm{D}}=+0.33(c 0.29$, $\left.\mathrm{CHCl}_{3}\right)$ ) (Scheme 3). ${ }^{18,19}$

The reaction should involve the regioselective cis-1,2-addition of the in situ generated phenylrhodium(I) species across the carbon-carbon triple bond, which should be directed through the dual coordinative interaction of rhodium with alkynyl and acetate moieties (I), to afford the alkenylrhodium(I) intermediate (II) (Scheme 3). ${ }^{7,8}$ It should be noted that the application of the rhodium-catalyzed protocol on enynes 4 and 5 , which lack a leaving group at the allylic carbon, resulted in complex mixtures of various isomers of mono- and diarylated products.
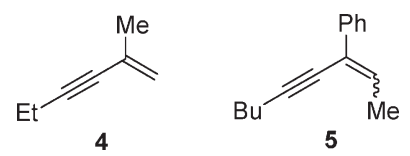

The subsequent $\delta$-elimination of [Rh]-OAc and double bond migration may take place concurrently via both anti and syn modes, ${ }^{20}$ syn-deacetylation being the major path, to produce the enantiomers of arylated vinylallene products and regenerating the active $\mathrm{Rh}(\mathrm{I})$ catalyst for the next cycle. Alternatively, the formation of a benzylidene- $\pi$-allylrhodium intermediate (III) may precede the deacetylation step. ${ }^{12,19}$

However, in both instances, the elimination step should be activated via the coordination of the rhodium and the oxygen functionality. ${ }^{20}$ Indeed, we found that the Rh-mediated protocol is not applicable to $(E)$-configured enyne acetates; an analogous reaction with $(E)$-1.1a furnished a dienyl acetate structure $(6)$ as the major product (62\% NMR yield), and consequently, formation of the desired vinylallene product was less than $10 \%$ (Scheme 4). This result indicates that the alkenylrhodium intermediate $\mathbf{I I}^{\prime}$, which is formed via rhodoarylation of $(E)$ 1.1a, could not undergo the $\delta$-elimination of $[\mathrm{Rh}]$-OAc, preferring instead demetalation to yield a hydroarylated product (6). ${ }^{7}$ The intermediate II' $^{\prime}$ cannot maintain a suitable geometry that is amenable to interaction with $\mathrm{Rh}(\mathrm{I})-\mathrm{OAc}$, this being a suspected prerequisite to the elimination step.

For better rationalization of the observed enantioselective outcome of the rhodium-mediated reaction of $(R, Z)-1.1$ a with phenylboronic acid, we performed conformational analyses by carrying out density functional theory (DFT) calculations for intermediate II. The conformations were investigated by considering different combinations of rotations about four different single carbon-carbon bonds $\left(\Theta_{1}-\Theta_{4}\right)$, as depicted in Figure 1, and 81 conformers were obtained. Then, more rotations were performed so as to achieve conformers that included the rhodium and the acetate groups in suitable relative proximity to one other for effective coordination (typically $2.239-2.286 \AA$ ), a necessary condition for the subsequent Rh-OAc elimination step leading to 3 .

The overall conformational analysis yielded a minimum energy that was associated with the conformer ES as shown in Figure 2. Interestingly, this conformer could potentially follow a $[\mathrm{Rh}]$-OAc elimination path leading to the product $(S)$-3aa. The other structures, which are assigned the identifiers ER, ZS, and ZR (Figure 2), represent the conformers of local minimum energies and potentially lead to the products $(E, R)-,(Z, S)$-, and $(Z, R)$-3aa, respectively. The fact that the conformer ER had a potential energy that is $4.1 \mathrm{kcal} / \mathrm{mol}$ higher than that of the conformer ES may account for the resulting enantiomeric ratio of the 3aa products. The relative energies of $\mathrm{ZR}$ and $\mathrm{ZS}$ as compared to ES are about 6.9 and $8.8 \mathrm{kcal} / \mathrm{mol}$ higher, respectively. It should be noted that no $(Z)$-configured vinylallene production was observed in the course of this study.

$\mathrm{Pd}(0)$-Catalyzed Reaction of 2-En-4-yne Carbonates and Organoborons. The catalyst system used for the $\operatorname{Pd}(0)$-catalyzed 
Scheme 3. Machanism and Stereochemistry of $\mathrm{Rh}(\mathrm{I})$-Catalyzed Arylation of $(R, Z)$-2-En-4-yne Acetate 1.1a

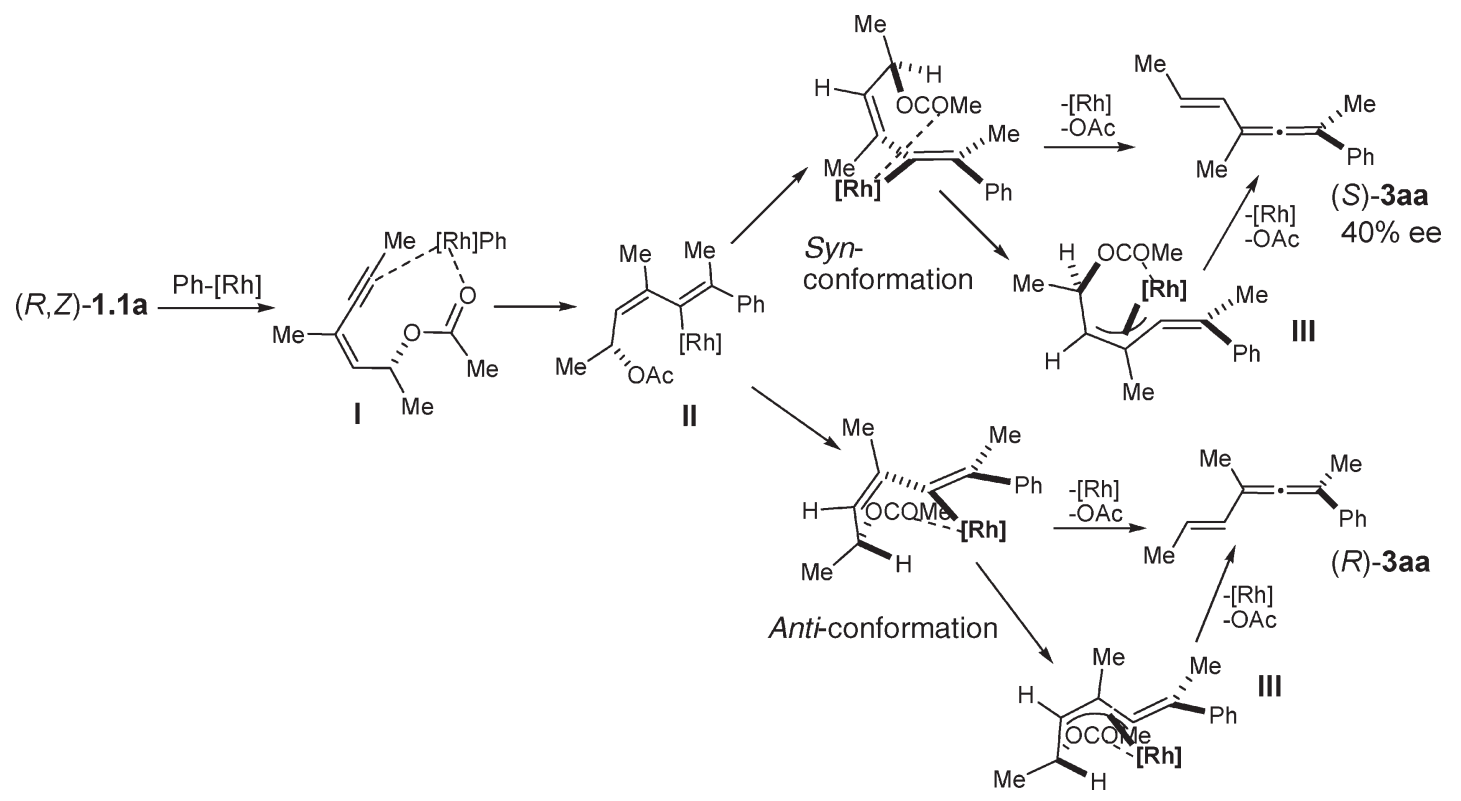

Scheme 4. $\mathrm{Rh}(\mathrm{I})$-Catalyzed Arylation of $(E)$-1.1a
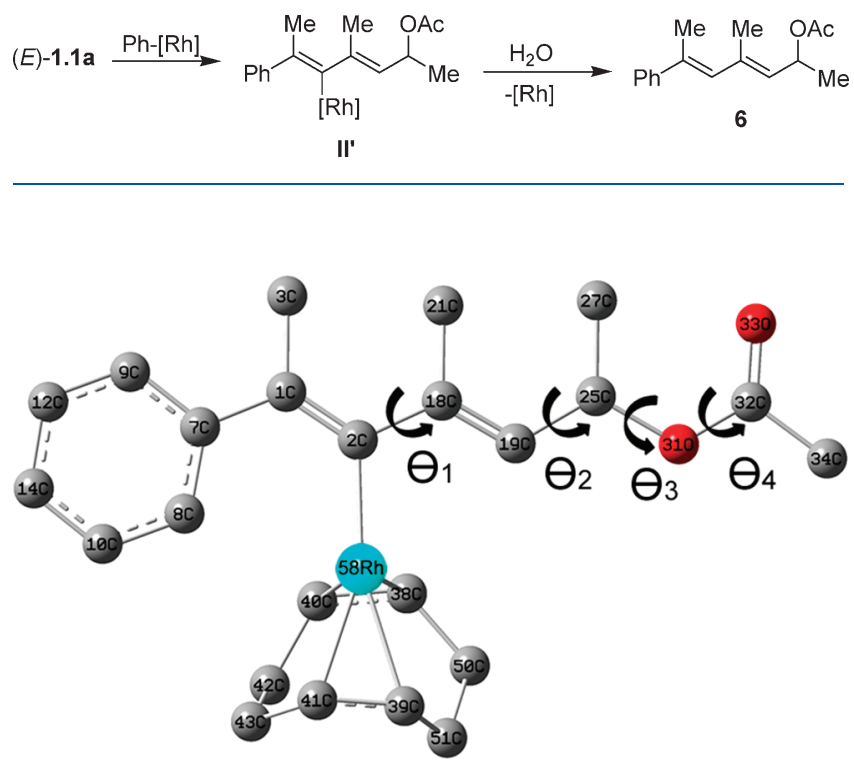

Figure 1. Rotational angles on the structure of intermediate II, which was used in conformational search (hydrogen atoms are omitted for clarity).

reactions of 2,4-enyne carbonates with organoboronic acids was $\mathrm{Pd}_{2}(\mathrm{dba})_{3} \mathrm{CHCl}_{3} / \mathrm{PPh}_{3}$, since this combination already proved its activity for $\mathrm{Pd}(0)$-catalyzed alkoxycarbonylation of conjugated $(Z)$ enyne carbonates, which led to vinylallenyl esters. ${ }^{13}$ Indeed, a related $(E)$-configured and phenyl-functionalized vinylallene 3aa was produced via the reaction of a $(Z)$-2-en-4-yne carbonate 1.2a with phenylboronic acid in the presence of $\mathrm{Pd}_{2}$ $(\mathrm{dba})_{3} \mathrm{CHCl}_{3}(3 \mathrm{~mol} \% \mathrm{Pd})$ and $\mathrm{PPh}_{3}(12 \mathrm{~mol} \%)$ in dry THF and at $65{ }^{\circ} \mathrm{C}$, albeit the reaction did not proceed to completion (Table 4, entry 1 ). The formation of 3aa dictates formation of a
$\sigma$-vinylallenylpalladium(II) intermediate and its subsequent transmetalation with the organoboronic acid (Schemes 2 and 5).

The presence of small amounts of water seems beneficial for the reaction; with its presence, the reaction of the enyne carbonate $(Z)$-1.2a with phenylboronic acid proceeded with complete conversion to give the 3 aa in high yields (entries 2 and 3). The application of a lower reaction temperature $\left(50^{\circ} \mathrm{C}\right)$ significantly reduced the efficacy of the process (entry 4 ), and the method can also be run at a lower Pd loading ( 2 mol \%) (entry 5 ).

The scope of the $\mathrm{Pd}(0)$-catalyzed method appears to be remarkably wider for both organoboronic acid and enyne carbonate substrate partners as compared to the $\mathrm{Rh}(\mathrm{I})$-catalyzed version. The reaction of $(Z)-\mathbf{1 . 2 a}$ with the highly electrondeficient $p-\mathrm{CF}_{3}(\mathbf{2 b})$ or 3,4-difluoro substituted phenylboronic $(2 \mathbf{j})$ acids and 3-pyridylboronic acid (2k) afforded moderate yields of vinylallene products (Table 5, entries $1-3$ ). Nevertheless the reactions with ortho-, para-, and meta-substituted electron-rich and moderately electron-poor phenylboronic acids, 1- and 2-naphthylboronic acids ( $\mathbf{2 m}$ and $\mathbf{2 h}$, respectively), as well as 3-thienylboronic acid (2i) provided the corresponding arylated vinylallene products at yields ranging between $73 \%$ and 92\% within relatively short reaction times (typically $1 \mathrm{~h}$ ) (entries 4-11). In contrast to the $\mathrm{Rh}(\mathrm{I})$-catalyzed version, alkenylboronic acids also proved to be an applicable class of organoboron reagents for the proposed $\operatorname{Pd}(0)$-catalyzed method. A divinylallene structure 3 an was produced at a yield of $74 \%$ when $(Z)-1.2 a$ reacted with 1-pentenylboronic acid for $1 \mathrm{~h}$ (entry 12).

The methodology can tolerate without problem phenyl $((Z)$ 1.2h), butyl $((Z)-\mathbf{1 . 2 b})$, cyclohexyl $((Z)-\mathbf{1 . 2 c})$, as well as highly bulky tert-butyl $((Z)-\mathbf{1 . 2 i})$ groups on the alkynyl terminus of the enyne carbonates $\left(R^{1}\right.$, Table 6 , entries $\left.1-4\right)$. Their reaction with phenylboronic acid under the optimal conditions provided the corresponding vinylallenes in good to high yields (58-88\%). A moderate yield ( $50 \%$, entry 5 ) of the vinylallene $3 \mathrm{ja}$ was produced when $\mathrm{R}^{1}$ is $\mathrm{H}((Z)-\mathbf{1 . 2} \mathrm{j})$. The product $3 \mathbf{j a}$, nonetheless, was formed at a higher yield ( $82 \%$, entry 6$)$ from the reaction of the $(Z)$-enyne carbonate where $\mathrm{R}^{1}$ was a $\mathrm{SiMe}_{3}$ group $((Z)-\mathbf{1 . 2 k})$ 

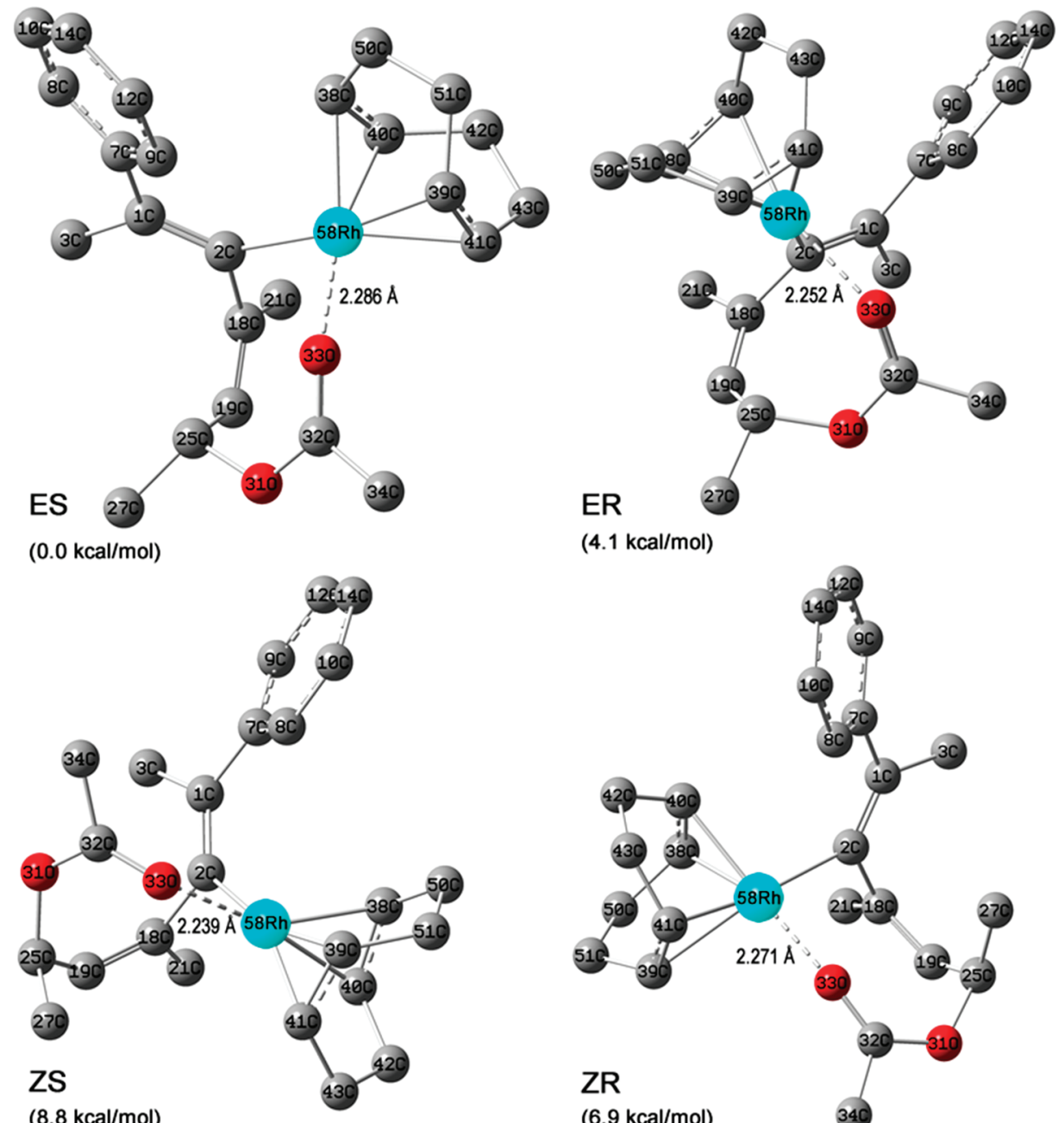

$(4.1 \mathrm{kcal} / \mathrm{mol})$

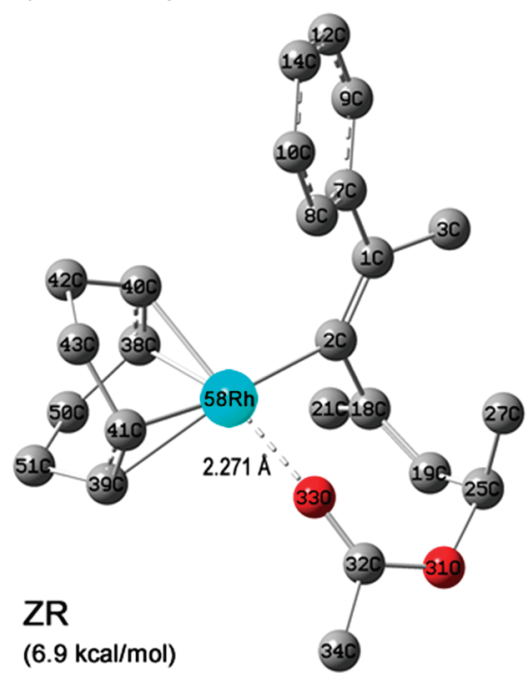

Figure 2. Optimized conformers for the intermediate II (hydrogen atoms are omitted for clarity and the energy values are relative energies).

Table 4. Effect of Reaction Conditions on the $\operatorname{Pd}(0)$-Catalyzed Reaction of the $(Z)$-Enyne Carbonate 1.2a with Phenylboronic Acid

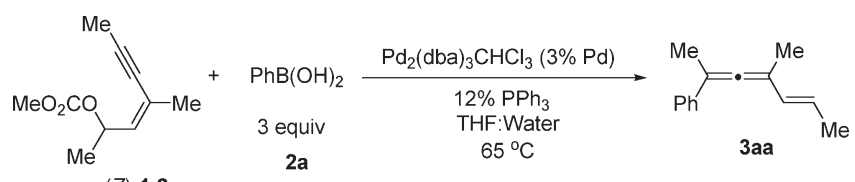
(Z)-1.2a 2a

$\begin{array}{cc} & \\ \text { version (\%) } & \text { yield (\%) } \\ 71 & 67 \\ 100 & 89 \\ 100 & (84)^{b} \\ 50 & 43 \\ 100 & 85\end{array}$

Determined by ${ }^{1} \mathrm{H}$ NMR using benzaldehyde as internal standard. ${ }^{b}$ Isolated yield. ${ }^{c} 50{ }^{\circ} \mathrm{C} .{ }^{d} 2 \% \mathrm{Pd}$ and $8 \% \mathrm{PPh}_{3}$.

upon the involvement of a desilylation process (entry 6). ${ }^{21}$ Substituting the methyl group in the $\mathrm{R}^{2}$ position with a butyl
Scheme 5. Transmetalation of $\boldsymbol{\sigma}$-Vinylallenylpalladium(II) and Organoboronic Acid<smiles>[R]C=CC([R])=C([R])[R]</smiles>

group $((Z)-1.2 d)$ had no significant effect on the activity of the enyne carbonate substrate ( $80 \%$ yield, entry 7 ); however, the corresponding vinylallene yields were relatively lower when $\mathrm{R}^{2}$ was $\mathrm{H}((Z)-\mathbf{1 . 2 l})$ or a phenyl group $((Z)-\mathbf{1 . 2 e})(61 \%$ and $64 \%$, entries 8 and 9 , respectively).

The effect of variation of allylic substitution on the activity of the enyne carbonate was also assessed. The method was rather successful also for the enyne substrates in which $\mathrm{R}^{3}$ was butyl ((Z)-1.2f, 76\%) or isopropyl ((Z)-1.2g, 92\%) groups; however, an enyne carbonate of a primary alcohol $\left(\mathrm{R}^{3}=\mathrm{H}\right)$ led to a low yield of $(44 \%)$ of vinylallene product $3 \mathrm{ma}$ (entries $10-12)$.

In contrast to the $\mathrm{Rh}$-mediated procedure, the $\mathrm{Pd}$-catalyzed method was also successful when used with $(E)$-configured 2,4enyne carbonates (Table 7); (E)-1.2a afforded the vinylallene product 3 aa in a yield of $69 \%$, which is somewhat lower than that 
Table 5. $\operatorname{Pd}(0)$-Catalyzed Reaction of the $(Z)$-Enyne Carbonate 1.2a with Organoboronic Acids

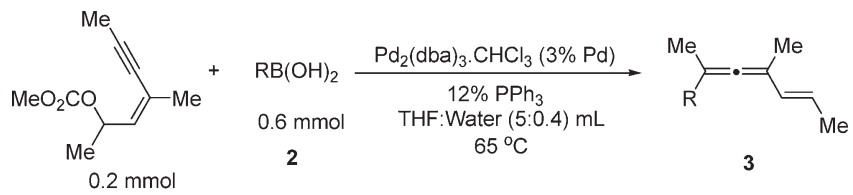

(Z)-1.2a

\begin{tabular}{|c|c|c|c|c|}
\hline entry & $\mathrm{R}$ & time $(\mathrm{h})$ & product & yield (\%) \\
\hline 1 & 2b $4-\mathrm{CF}_{3} \mathrm{C}_{6} \mathrm{H}_{4}$ & 8 & $3 a b$ & 56 \\
\hline 2 & $2 \mathrm{j} 3,4-\mathrm{F}_{2} \mathrm{C}_{6} \mathrm{H}_{4}$ & 24 & 3aj & 58 \\
\hline 3 & 2k 3-pyridyl & 6 & 3ak & 59 \\
\hline 4 & $2 \mathrm{c} 4-\mathrm{MeCOC}_{6} \mathrm{H}_{4}$ & 2 & $3 \mathrm{ac}$ & 79 \\
\hline 5 & $2 \mathrm{~d} 4-\mathrm{MeC}_{6} \mathrm{H}_{4}$ & 1 & $3 \mathrm{ad}$ & 92 \\
\hline 6 & 2e $3-\mathrm{MeC}_{6} \mathrm{H}_{4}$ & 1 & $3 a e$ & 80 \\
\hline 7 & $2 \mathrm{~g} 2-\mathrm{MeOC}_{6} \mathrm{H}_{4}$ & 1 & $3 a g$ & 88 \\
\hline 8 & $212-\mathrm{FC}_{6} \mathrm{H}_{4}$ & 1 & 3al & 82 \\
\hline 9 & 2h 2-naphthyl & 1 & $3 \mathrm{ah}$ & 75 \\
\hline 10 & 2m 1-naphthyl & 1 & 3am & 83 \\
\hline 11 & 2i 3-thienyl & 1 & 3ai & 73 \\
\hline 12 & 2n 1-pentenyl & 1 & 3 an & 74 \\
\hline
\end{tabular}

Table 6. Pd(0)-Catalyzed Reaction of $(Z)$-Enyne Carbonates with Phenylboronic Acid

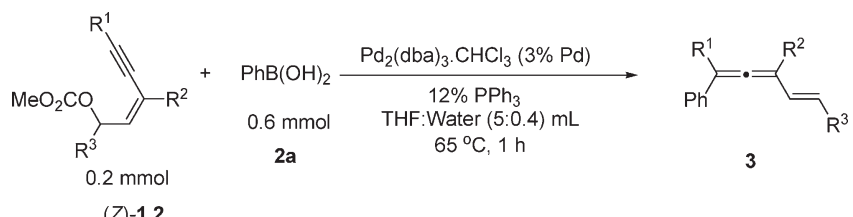

(Z)-1.2

\begin{tabular}{|c|c|c|c|c|c|c|}
\hline entry & substrate & $\mathrm{R}^{1}$ & $\mathrm{R}^{2}$ & $\mathrm{R}^{3}$ & product & yield (\%) \\
\hline 1 & $(Z)-1.2 \mathrm{~h}$ & $\mathrm{Ph}$ & $\mathrm{Me}$ & $\mathrm{Me}$ & 3ha & 88 \\
\hline 2 & $(Z)-\mathbf{1 . 2 b}$ & $\mathrm{Bu}$ & $\mathrm{Me}$ & $\mathrm{Me}$ & $3 \mathrm{ba}$ & 82 \\
\hline 3 & $(Z)-1.2 \mathrm{c}$ & Cy & $\mathrm{Me}$ & $\mathrm{Me}$ & $3 \mathrm{ca}$ & 67 \\
\hline $4^{a}$ & $(Z)-1.2 \mathrm{i}$ & $t-B u$ & $\mathrm{Me}$ & $\mathrm{Me}$ & $3 i a$ & 58 \\
\hline 5 & $(Z)-1.2 \mathbf{j}$ & $\mathrm{H}$ & $\mathrm{Me}$ & $\mathrm{Me}$ & $3 \mathrm{ja}$ & 50 \\
\hline 6 & $(Z)-1.2 \mathrm{k}$ & $\mathrm{Me}_{3} \mathrm{Si}$ & $\mathrm{Me}$ & $\mathrm{Me}$ & $3 \mathrm{ja}$ & $82\left(\mathrm{R}^{1}=\mathrm{H}\right)^{b}$ \\
\hline 7 & $(Z)-1.2 \mathrm{~d}$ & $\mathrm{Bu}$ & $\mathrm{Bu}$ & $\mathrm{Me}$ & $3 \mathrm{da}$ & 80 \\
\hline 8 & $(Z)-1.21$ & $\mathrm{Bu}$ & $\mathrm{H}$ & $\mathrm{Me}$ & 3la & 61 \\
\hline 9 & $(Z)-1.2 \mathrm{e}$ & $\mathrm{Bu}$ & $\mathrm{Ph}$ & $\mathrm{Me}$ & $3 e a$ & 64 \\
\hline $10^{c}$ & $(Z)-1.2 f$ & $\mathrm{Me}$ & $\mathrm{Me}$ & $\mathrm{Bu}$ & $3 \mathrm{fa}$ & 76 \\
\hline 11 & $(Z)-1.2 \mathrm{~g}$ & $\mathrm{Me}$ & $\mathrm{Me}$ & $i-\operatorname{Pr}$ & $3 g a$ & 92 \\
\hline 12 & $(Z)-1.2 \mathrm{~m}$ & $\mathrm{Bu}$ & $\mathrm{Me}$ & $\mathrm{H}$ & $3 \mathrm{ma}$ & 44 \\
\hline
\end{tabular}

obtained from its $(Z)$-configured isomer when reacted with phenylboronic acid at $65^{\circ} \mathrm{C}$ for $1 \mathrm{~h}$ (entry 1 ). Nevertheless, in contrast with the reactivity of $(Z)-1.2 \mathrm{a}$, the same yield could also be achieved at $50{ }^{\circ} \mathrm{C}$ (entry 2); however, at this lower reaction temperature, the conversion was incomplete when using $(Z)$ 1.2a (see Table 4, entry 4). The activity of the Pd-catalysis, however, was insufficient at room temperature; the conversion was low and consequently gave rise to a small yield of the desired vinylallene product (entry 3 ).
Table 7. $\operatorname{Pd}(0)$-Catalyzed Reaction of the (E)-Enyne Carbonate 1.2a with Organoboronic Acids

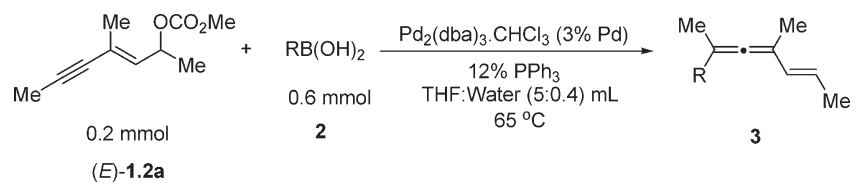

\begin{tabular}{|c|c|c|c|c|}
\hline entry & $\mathrm{R}$ & time $(\mathrm{h})$ & product & yield (\%) \\
\hline 1 & $2 \mathrm{aPh}$ & 1 & 3aa & 69 \\
\hline $2^{a}$ & & 1 & & 69 \\
\hline $3^{b}$ & & 6 & & 25 \\
\hline 4 & $2 \mathbf{b} 4-\mathrm{CF}_{3} \mathrm{C}_{6} \mathrm{H}_{4}$ & 5 & $3 a b$ & 61 \\
\hline 5 & 2c $4-\mathrm{MeCOC}_{6} \mathrm{H}_{4}$ & 10 & $3 a c$ & 81 \\
\hline 6 & 2d 4- $\mathrm{MeC}_{6} \mathrm{H}_{4}$ & 1 & $3 a d$ & 77 \\
\hline 7 & 2e $3-\mathrm{MeC}_{6} \mathrm{H}_{4}$ & 1 & $3 a e$ & 76 \\
\hline 8 & $2 \mathrm{~g} 2-\mathrm{MeOC}_{6} \mathrm{H}_{4}$ & 1 & $3 a g$ & 75 \\
\hline 9 & $212-\mathrm{FC}_{6} \mathrm{H}_{4}$ & 1 & 3al & 77 \\
\hline 10 & 2m 1-naphthyl & 1 & 3am & 71 \\
\hline 11 & 2i 3-thienyl & 1 & $3 a i$ & 77 \\
\hline 12 & 2k 3-pyridyl & 10 & 3ak & 71 \\
\hline 13 & 2n 1-pentenyl & 1 & 3an & 74 \\
\hline
\end{tabular}

Table 8. $\operatorname{Pd}(0)$-Catalyzed Reaction of $(E)$-Enyne Carbonates with Phenylboronic Acid

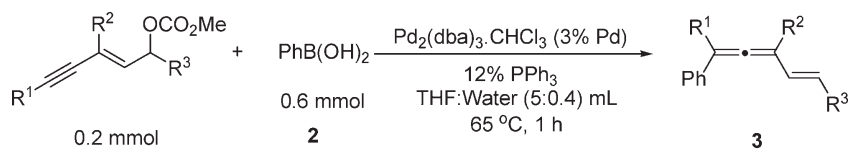

(E)-1.2

\begin{tabular}{ccccccc} 
entry & substrate & $\mathrm{R}^{1}$ & $\mathrm{R}^{2}$ & $\mathrm{R}^{3}$ & product & yield (\%) \\
1 & $(E)-\mathbf{1 . 2 b}$ & $\mathrm{Bu}$ & $\mathrm{Me}$ & $\mathrm{Me}$ & 3ba & 78 \\
2 & $(E)-\mathbf{1 . 2 h}$ & $\mathrm{Ph}$ & $\mathrm{Me}$ & $\mathrm{Me}$ & $3 \mathrm{ha}$ & 87 \\
3 & $(E)-\mathbf{1 . 2 f}$ & $\mathrm{Me}$ & $\mathrm{Me}$ & $\mathrm{Bu}$ & $3 \mathrm{fa}$ & 75 \\
4 & $(E)-\mathbf{1 . 2} \mathrm{g}$ & $\mathrm{Me}$ & $\mathrm{Me}$ & $i$-Pr & 3 ga & 83 \\
5 & $(E)-\mathbf{1 . 2 n}$ & $\mathrm{Me}$ & $\mathrm{H}$ & $\mathrm{Me}$ & 3 na & $<10$ \\
\hline
\end{tabular}

Scheme 6. Mechanism and Stereochemistry of $\operatorname{Pd}(0)$-Catalyzed Arylation of $(R, Z)$-2-En-4-yne Carbonate 1.2a

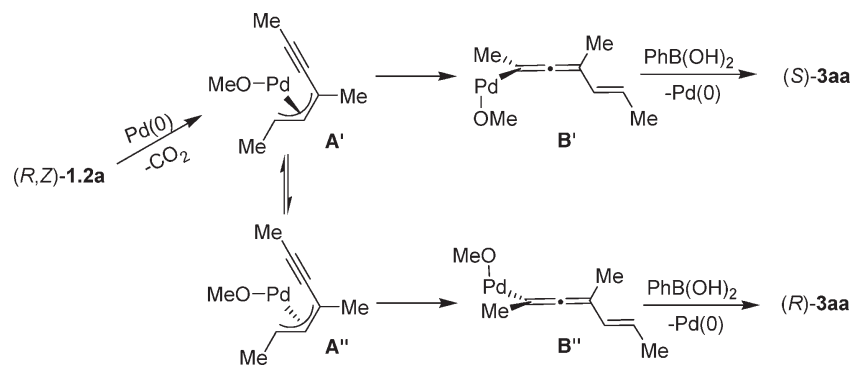

(E)-1.2a reacted with various electron-rich and electron-poor, as well as sterically encumbered phenylboronic acids successfully to produce the related vinylallenes in good yields (entries 4-9). 
1-Napthyl, heteroaryl, and alkenylboronic acids were also suitable components (entries 10-13).

The enyne reagents with butyl $((E)-\mathbf{1 . 2 b})$ or phenyl substituent on the alkynyl carbon $((E)-\mathbf{1 . h})$ and butyl $((E)-\mathbf{1 . 2 f})$ or isopropyl $((E)-\mathbf{1 . 2} \mathbf{g})$ groups on the allylic carbon all gave rise to high yields of the corresponding vinylallene products when reacted with phenylboronic acid (Table 8 , entries $1-4)$. Interestingly, however, the reaction of an $(E)$-enyne carbonate $((E)$ 1.2n), where $\mathrm{R}^{2}$ is $\mathrm{H}$, with phenylboronic acid, in contrast to its analogue $(Z)$-configured substrate $((Z)-\mathbf{1 . 2 1})$, gave rise to an intricate mixture of products, containing less than $10 \%$ of the corresponding phenylated vinylallene (entry 5).

No center-to-axis chirality transfer selectivity could be demonstrated with the existing method, as is the reported case for the $\operatorname{Pd}(0)$-catalyzed alkoxycarbonylation applications with these substrates. ${ }^{13}$ The relevant reaction of an enantiomerically enriched enyne carbonate $(R, Z)-\mathbf{1 . 2 a}(94.5 \%$ ee ) with phenylboronic acid led to a racemic mixture of the product.

The catalytic cycle should involve an oxidative cleavage leading to a $\pi$-allylpalladium intermediate $\left(\mathbf{A}^{\prime}\right)$ and subsequent isomerization of this intermediate to $\mathbf{A}^{\prime \prime}$ could be the basis for the observed racemization (Scheme 6). ${ }^{22}$ The shift of palladium to the distal alkynyl carbon yields a racemate of $3 \mathrm{aa}$.

\section{CONCLUSION}

In this report, the first examples of the transition-metalcatalyzed 1,5-substitution reactions of 2-en-4-yne reagents with a leaving group in the allylic position and organoboronic acids are presented. The reactions produced vinylallenes with an exclusively $(E)$-configuration in both $\mathrm{Rh}(\mathrm{I})$ - and $\mathrm{Pd}(0)$-catalyzed procedures. $\mathrm{Rh}(\mathrm{I})$-catalyzed reactions proceeded via typically $\mathrm{S}_{\mathrm{N}} 2^{\prime \prime}$-type substitution of (Z)-2-en-4-yne acetates, which is triggered by cis-addition of in situ formed nucleophilic arylrhodium(I) species. The $\delta$-elimination of $\mathrm{Rh}(\mathrm{I})$-OAc, which is promoted via coordinative interactions, took place in both syn and anti modes, with the syn-elimination being the dominant route. The $(E)$-configured enynes are not suitable reagents for the $\mathrm{Rh}(\mathrm{I})$-catalyzed method, since geometrical constraints prohibit the required coordinative interactions. The $\mathrm{Pd}(0)$-catalyzed method can be employed for conjugated enynes that contain a carbonate functionality in the allylic position and is applicable for a wider range of organoboronic acids and both (E)- and (Z)configured enyne substrates. For an enantiomerically enriched (Z)-enyne carbonate, the $\operatorname{Pd}(0)$-catalyzed method led to complete racemization, in contrast to the $\mathrm{Rh}(\mathrm{I})$-catalyzed method.

\section{EXPERIMENTAL SECTION}

General. The synthesized reactants and vinylallene products were analyzed by GC and GC-MS and isolated by column chromatography using a hexane/ethyl acetate eluent. The vinylallene products are colorless or light yellow oil, and coupling constants of olefinic protons and NOE studies confirmed (E)-configured structures. NMR spectra were recorded within $\mathrm{CDCl}_{3}$ or $\mathrm{C}_{6} \mathrm{D}_{6}$ solvents. ${ }^{23}$ Infrared spectra were obtained by ATR method with neat samples. Enantiomeric excess values were determined by an HPLC method with an OD column $(25 \mathrm{~cm} \times$ $0.46 \mathrm{~cm})$ and using hexane eluent $(1 \mathrm{~mL} / \mathrm{min}$; racemic $(Z)$-3aa eluted at the retention times of $4.0(R)$ and $4.2(S)$ minutes). The $\mathrm{Pd}_{2^{-}}$ $(\mathrm{dba})_{3} \mathrm{CHCl}_{3},{ }^{24}[\mathrm{RhCl}(\mathrm{cod})]_{2},{ }^{25}$ and $[\mathrm{RhOH}(\mathrm{cod})]_{2}{ }^{26}$ complexes were synthesized in laboratory.

Preparation of the Enyne Substrates. The synthesis of enyne carbonate $(Z)-\mathbf{1 . 2 k}$ involved the Sonogashira coupling of ethynyltrimethylsilane with a corresponding vinyl iodide reagent as described elsewhere. ${ }^{13}$ The enyne carbonate containing a terminal alkynyl group $((Z)-\mathbf{1 . 2 j})$ was obtained via desilylation ${ }^{27}$ of a corresponding $\mathrm{Me}_{3} \mathrm{Si}$ substituted 2,4-enynol ((Z)-4-methyl-6-(trimethylsilyl)hex3-en-5-yn-2-ol) and the following conversion of the hydroxyl group to carbonate. The methods for the syntheses of all other $(Z)$-enyne carbonates are described elsewhere. ${ }^{13}$ Acetates and carbonates of $(E)$ 2-en-4-yn-1-ols were synthesized following the described methods starting from (E)-pent-2-en-4-yn-1-ol reagent. ${ }^{13}$ Racemic enyne acetates and $(S)$-1.1a were prepared by acetylation ${ }^{11 a}$ of the corresponding enynol reagents, which were synthesized as described previously. ${ }^{13}$ All substrates were colorless oils.

(Z)-1.1a. ${ }^{1} \mathrm{H}$ NMR (400 MHz, $\left.\mathrm{CDCl}_{3}\right) \delta 5.71(\mathrm{dq}, J=8.8,6.8 \mathrm{~Hz}$, $1 \mathrm{H}), 5.56(\mathrm{dq}, J=8.8,1.2 \mathrm{~Hz}, 1 \mathrm{H}), 2.01(\mathrm{~s}, 3 \mathrm{H}), 1.97(\mathrm{~s}, 3 \mathrm{H}), 1.81(\mathrm{~d}, J=$ $1.2 \mathrm{~Hz}, 3 \mathrm{H}), 1.28(\mathrm{~d}, J=6.8 \mathrm{~Hz}, 3 \mathrm{H}) ;{ }^{13} \mathrm{C} \mathrm{NMR}\left(101 \mathrm{MHz}, \mathrm{CDCl}_{3}\right) \delta$ $170.4,134.9,121.5,91.7,77.7,70.3,23.5,21.5,20.5,4.5$; IR ( $v_{\max } /$ $\left.\mathrm{cm}^{-1}\right)$ 2982, 2233, 1730, 1639, 1433, 1235, 1152, 1046; MS (EI, $\left.m / z\right)$ $166\left(11, \mathrm{M}^{+}\right), 151$ (37), 137 (4), 123 (100), 109 (92), 91 (98), 79 (33); HRMS (EI) calcd for $\mathrm{C}_{10} \mathrm{H}_{14} \mathrm{O}_{2}\left(\mathrm{M}^{+}\right)$166.0988, found 166.0993 .<smiles>CC#C/C(C)=C/C(C)OC(C)=O</smiles>

(Z)-1.1b. ${ }^{1} \mathrm{H}$ NMR (400 MHz, $\left.\mathrm{CDCl}_{3}\right) \delta 5.73(\mathrm{dq}, J=8.4,6.4 \mathrm{~Hz}$, $1 \mathrm{H}), 5.56(\mathrm{dq}, J=8.4,1.4 \mathrm{~Hz}, 1 \mathrm{H}), 2.34(\mathrm{t}, J=7.0 \mathrm{~Hz}, 2 \mathrm{H}), 2.02(\mathrm{~s}, 3 \mathrm{H})$, $1.83(\mathrm{~d}, J=1.6 \mathrm{~Hz}, 3 \mathrm{H}), 1.38-1.58(\mathrm{~m}, 4 \mathrm{H}), 1.29(\mathrm{~d}, J=6.4 \mathrm{~Hz}, 3 \mathrm{H}), 0.92$ $(\mathrm{t}, J=7.2 \mathrm{~Hz}, 3 \mathrm{H}) ;{ }^{13} \mathrm{C} \mathrm{NMR}\left(101 \mathrm{MHz}, \mathrm{CDCl}_{3}\right) \delta 170.3,134.9,121.6$, 96.4, 78.6, 70.4, 30.9, 23.6, 22.1, 21.5, 20.4, 19.3, 13.7; IR $\left(\nu_{\max } / \mathrm{cm}^{-1}\right) 2941$, 2870, 2223, 1736, 1636, 1371, 1232, 1036; MS (EI, $m / z) 208\left(8, \mathrm{M}^{+}\right), 193$ (8), 179 (4), 165 (100), 151 (24), 137 (14), 123 (49), 109 (88), 91 (82), 79 (47); HRMS (EI) calcd for $\mathrm{C}_{13} \mathrm{H}_{20} \mathrm{O}_{2}\left(\mathrm{M}^{+}\right)$208.1458, found 208.1455 .<smiles>CC(=O)OC(C)/C=C(/C)C#CBr</smiles>

(Z)-1.1c. ${ }^{1} \mathrm{H}$ NMR (400 MHz, $\left.\mathrm{CDCl}_{3}\right) \delta 5.78-5.66(\mathrm{~m}, 1 \mathrm{H}), 5.56$ $(\mathrm{dq}, J=8.4,0.8 \mathrm{~Hz}, 2 \mathrm{H}), 2.55-2.48(\mathrm{~m}, 1 \mathrm{H}), 2.01(\mathrm{~s}, 3 \mathrm{H}), 1.82(\mathrm{~d}, J=$ $1.2 \mathrm{~Hz}, 3 \mathrm{H}), 1.83-1.76(\mathrm{~m}, 2 \mathrm{H}), 1.66-1.74(\mathrm{~m}, 2 \mathrm{H}), 1.43-1.53(\mathrm{~m}$, $3 \mathrm{H}), 1.30-1.37(\mathrm{~m}, 3 \mathrm{H}), 1.35(\mathrm{~d}, J=5.6 \mathrm{~Hz}, 3 \mathrm{H}) ;{ }^{13} \mathrm{C}$ NMR $(100$ $\left.\mathrm{MHz}_{\mathrm{CDCl}}\right) \delta 170.3,134.8,128.6,121.8,100.5,78.5,70.3,63.4,32.8$, 29.8, 26.0, 24.9, 23.6, 21.5, 20.4; IR $\left(v_{\max } / \mathrm{cm}^{-1}\right)$ 2971, 2929, 2854, 2212, 1737, 1448, 1368, 1234, 1153, 1041; MS (EI, $m / z) 234\left(10, \mathrm{M}^{+}\right)$, 219 (12), 191 (89), 177 (19), 149 (26), 131 (31), 117 (24), 109 (100), 91 (51), 77 (25); HRMS (EI) calcd for $\mathrm{C}_{15} \mathrm{H}_{22} \mathrm{O}_{2}\left(\mathrm{M}^{+}\right)$234.1614, found 234.1616 .

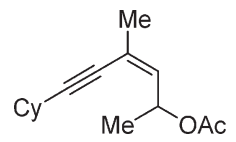

(Z)-1.1d. ${ }^{1} \mathrm{H}$ NMR (400 MHz, $\left.\mathrm{C}_{6} \mathrm{D}_{6}\right) \delta 6.25(\mathrm{dq}, J=8.4,6.4 \mathrm{~Hz}$, $1 \mathrm{H}), 5.67(\mathrm{~d}, J=8.8 \mathrm{~Hz}, 1 \mathrm{H}), 2.15(\mathrm{t}, J=6.8 \mathrm{~Hz}, 2 \mathrm{H}), 2.11(\mathrm{t}, J=8.0 \mathrm{~Hz}$, $2 \mathrm{H}), 1.69(\mathrm{~s}, 3 \mathrm{H}), 1.56$ (quint, $J=7.6 \mathrm{~Hz}, 2 \mathrm{H}), 1.42-1.31(\mathrm{~m}, 4 \mathrm{H}), 1.38$ $(\mathrm{d}, J=6.4 \mathrm{~Hz}, 3 \mathrm{H}), 1.30-1.20(\mathrm{~m}, 2 \mathrm{H}), 0.85(\mathrm{t}, J=7.2 \mathrm{~Hz}, 3 \mathrm{H}), 0.81(\mathrm{t}, J$ $=7.2 \mathrm{~Hz}, 3 \mathrm{H}) ;{ }^{13} \mathrm{C} \mathrm{NMR}\left(101 \mathrm{MHz}, \mathrm{C}_{6} \mathrm{D}_{6}\right) \delta 169.4,135.1,126.7,97.1$, 78.5, 70.1, 37.4, 31.1, 30.8, 22.4, 22.2, 21.0, 20.7, 19.4, 14.1, 13.7; IR $\left(v_{\max } / \mathrm{cm}^{-1}\right) 2955,2932,2865,2216,1742,1631,1457,1369,1237$, 1041; MS (EI, $m / z) 250\left(3, \mathrm{M}^{+}\right) 235$ (10), 221 (3), 207 (80), 190 (28), 161 (33), 151 (68), 133 (48), 119 (51), 105 (100), 91 (98), 77 (34); HRMS (EI) calcd for $\mathrm{C}_{16} \mathrm{H}_{26} \mathrm{O}_{2}\left(\mathrm{M}^{+}\right)$250.1927, found 250.1928 .

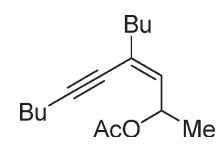


(Z)-1.1e. ${ }^{1} \mathrm{H}$ NMR (400 MHz, $\left.\mathrm{C}_{6} \mathrm{D}_{6}\right) \delta 7.70-7.65$ (m, 2H), 7.17$7.04(\mathrm{~m}, 3 \mathrm{H}), 6.42\left(\mathrm{dq}, J_{\mathrm{AB}}=8.4,6.4 \mathrm{~Hz}, 1 \mathrm{H}\right), 6.34\left(\mathrm{~d}, J_{\mathrm{AB}}=8.4 \mathrm{~Hz}, 1 \mathrm{H}\right)$, $2.18(\mathrm{t}, J=6.8 \mathrm{~Hz}, 2 \mathrm{H}), 1.70(\mathrm{~s}, 3 \mathrm{H}), 1.44-1.29(\mathrm{~m}, 4 \mathrm{H}), 1.42(\mathrm{~d}, J=6.4$ $\mathrm{Hz}, 3 \mathrm{H}), 0.81(\mathrm{t}, J=7.2 \mathrm{~Hz}, 3 \mathrm{H}) ;{ }^{13} \mathrm{C}$ NMR $\left(101 \mathrm{MHz}, \mathrm{C}_{6} \mathrm{D}_{6}\right) \delta 169.4$, 138.2, 135.3, 128.7, 128.4, 126.7, 126.2, 99.2, 77.4, 70.4, 31.0, 22.3, 20.9, 20.5, 19.5, 13.7; IR $\left(v_{\max } / \mathrm{cm}^{-1}\right) 2960,2932,2865,2233,1739,1368,1234$, 1039, 761, 693; MS (EI, $m / z) 270\left(6, \mathrm{M}^{+}\right) 255(6), 227(63), 211$ (16), 185 (42), 167 (100), 153 (94), 141 (33), 128 (34), 115 (37), 91 (23), 77 (14); HRMS (EI) calcd for $\mathrm{C}_{18} \mathrm{H}_{22} \mathrm{O}_{2}\left(\mathrm{M}^{+}\right)$270.1614, found 270.1613 .

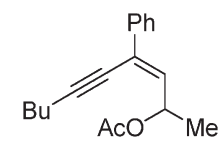

(Z)-1.1f. ${ }^{1} \mathrm{H} \mathrm{NMR}\left(400 \mathrm{MHz}, \mathrm{C}_{6} \mathrm{D}_{6}\right) \delta 6.12(\mathrm{dt}, J=8.4,6.8 \mathrm{~Hz}, 1 \mathrm{H})$, $5.55(\mathrm{dq}, J=8.6,1.0 \mathrm{~Hz}, 1 \mathrm{H}), 1.83-1.74(\mathrm{~m}, 2 \mathrm{H}), 1.76(\mathrm{~d}, J=1.2 \mathrm{~Hz}$, $3 \mathrm{H}), 1.72(\mathrm{~s}, 3 \mathrm{H}), 1.60(\mathrm{~s}, 3 \mathrm{H}), 1.38-1.25(\mathrm{~m}, 4 \mathrm{H}), 0.84(\mathrm{t}, J=7.2 \mathrm{~Hz}$, $3 \mathrm{H}) ;{ }^{13} \mathrm{C} \mathrm{NMR}\left(101 \mathrm{MHz}, \mathrm{C}_{6} \mathrm{D}_{6}\right) \delta 169.4,134.7,122.6,91.8,78.6,73.3$, 34.9, 27.6, 23.6, 22.9, 20.9, 14.2, 4.05; IR $\left(\nu_{\max } / \mathrm{cm}^{-1}\right) 2958,2922,2857$, 2232, 1738, 1634, 1363, 1371, 1234, 1018, 953; MS (EI, $m / z) 208$ (5, $\mathrm{M}^{+}$), 193 (14), 165 (59), 151 (43), 123 (28), 109 (100), 91 (49), 79 (32); HRMS (EI) calcd for $\mathrm{C}_{13} \mathrm{H}_{20} \mathrm{O}_{2}\left(\mathrm{M}^{+}\right)$208.1458, found 208.1456.<smiles>CC#CC(C)=CC(CC)OC(C)=O</smiles>

(Z)-1.1g. ${ }^{1} \mathrm{H}$ NMR (400 MHz, $\left.\mathrm{C}_{6} \mathrm{D}_{6}\right) \delta 6.01(\mathrm{dd}, J=8.8,6.4 \mathrm{~Hz}$, $1 \mathrm{H}), 5.55(\mathrm{dq}, J=8.8,0.8 \mathrm{~Hz}, 1 \mathrm{H}), 1.98$ (octet, $J=6.8 \mathrm{~Hz}, 1 \mathrm{H}), 1.77$ (d, $J=1.6 \mathrm{~Hz}, 3 \mathrm{H}), 1.72(\mathrm{~s}, 3 \mathrm{H}), 1.60(\mathrm{~s}, 3 \mathrm{H}), 0.98$ (d, $J=6.8 \mathrm{~Hz}, 3 \mathrm{H}), 0.95$ $(\mathrm{d}, J=6.8 \mathrm{~Hz}, 3 \mathrm{H}) ;{ }^{13} \mathrm{C} \mathrm{NMR}\left(101 \mathrm{MHz}, \mathrm{C}_{6} \mathrm{D}_{6}\right) \delta 169.3,132.7,123.6$, 91.7, 78.8, 77.4, 33.0, 23.8, 20.8, 18.4, 18.1, 4.10; IR $\left(v_{\max } / \mathrm{cm}^{-1}\right)$ 2963, 2920, 2876, 2240, 1733, 1637, 1435, 1369, 1232, 1017, 972, 606; MS (EI, $m / z) 194\left(4, \mathrm{M}^{+}\right), 179(2), 151$ (41), 137 (8), 119 (24), 109 (100), 91 (15), 79 (13); HRMS (EI) calcd for $\mathrm{C}_{12} \mathrm{H}_{18} \mathrm{O}_{2}\left(\mathrm{M}^{+}\right)$194.1301, found 194.1307.

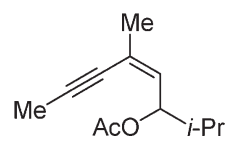

(Z)-1.2j. ${ }^{1} \mathrm{H}$ NMR (400 MHz, $\left.\mathrm{C}_{6} \mathrm{D}_{6}\right) \delta(\mathrm{dq}, J=8.0,6.5 \mathrm{~Hz}, 1 \mathrm{H}), 5.56$ $(\mathrm{d}, J=8.0 \mathrm{~Hz}, 1 \mathrm{H}), 3.34(\mathrm{~s}, 3 \mathrm{H}), 2.80(\mathrm{~s}, 1 \mathrm{H}), 1.58(\mathrm{~d}, J=1.2 \mathrm{~Hz}, 3 \mathrm{H})$, $1.26(\mathrm{~d}, J=6.4 \mathrm{~Hz}, 3 \mathrm{H}) ;{ }^{13} \mathrm{C}$ NMR $\left(101 \mathrm{MHz}, \mathrm{C}_{6} \mathrm{D}_{6}\right) \delta$ 155.6, 138.0, 120.4, 83.4, 81.4, 73.8, 54.1, 22.7, 20.2; FTIR $\left(v_{\max } / \mathrm{cm}^{-1}\right)$ 2957, 2931, 2860, 2244, 146 1441, 1258, 1033, 944, 791, 762, 697; MS (EI, $m / z) 168$ (2, $\left.\mathrm{M}^{+}\right), 153(4), 109(100), 91(77), 77(44)$; HRMS (EI) calcd for $\mathrm{C}_{9} \mathrm{H}_{12} \mathrm{O}_{3}\left(\mathrm{M}^{+}\right)$168.0781, found 168.0780 .

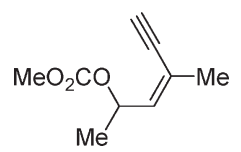

(Z)-1.2k. ${ }^{1} \mathrm{H}$ NMR (400 MHz, $\left.\mathrm{CDCl}_{3}\right) \delta 5.70\left(\mathrm{dq}, J_{\mathrm{AB}}=8.8\right.$, $1.6 \mathrm{~Hz}, 1 \mathrm{H}), 5.62(\mathrm{dq}, J=6.4,1.2 \mathrm{~Hz}, 1 \mathrm{H}), 3.76(\mathrm{~s}, 3 \mathrm{H}), 1.85(\mathrm{~d}, J=$ $0.8 \mathrm{~Hz}, 3 \mathrm{H}), 1.37(\mathrm{~d}, J=6.8 \mathrm{~Hz}, 3 \mathrm{H}), 0.2(\mathrm{~s}, 9 \mathrm{H}) ;{ }^{13} \mathrm{C} \mathrm{NMR}$ $\left(100 \mathrm{MHz}, \mathrm{CDCl}_{3}\right) \delta 155.1,136.9,121.4,102.7,100.5,74.1,54.7$, 23.0, 20.1, -0.01; FTIR $\left(v_{\max } / \mathrm{cm}^{-1}\right) 2958,2147,1747,1442,1254$, 1036, 880, 837, 791, 760, 634; MS (EI, $m / z) 240\left(12, \mathrm{M}^{+}\right), 225(3)$, 197 (3), 181 (100), 165 (35), 149 (88), 136 (13), 121 (22), 107 (12), 97 (23), 89 (64), 73 (63), 59 (33); HRMS (EI) calcd for $\mathrm{C}_{12} \mathrm{H}_{20} \mathrm{O}_{3} \mathrm{Si}$ $\left(\mathrm{M}^{+}\right)$240.1176, found 240.1165.

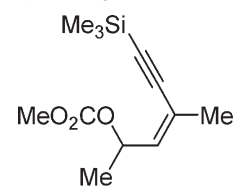

(E)-1.1a. ${ }^{1} \mathrm{H}$ NMR $\left(400 \mathrm{MHz}, \mathrm{CDCl}_{3}\right) \delta 5.65\left(\mathrm{~d}, J_{\mathrm{AB}}=9.2,1 \mathrm{H}\right)$, $5.54-5.61\left(\mathrm{dq}, J_{\mathrm{AB}}=8.8,6.4 \mathrm{~Hz}, 1 \mathrm{H}\right), 2.01(\mathrm{~s}, 3 \mathrm{H}), 1.93(\mathrm{~s}, 3 \mathrm{H}), 1.85(\mathrm{~d}$, $J=0.8 \mathrm{~Hz}, 3 \mathrm{H}), 1.27(\mathrm{~d}, J=6.4 \mathrm{~Hz}, 3 \mathrm{H}) ;{ }^{13} \mathrm{C} \mathrm{NMR}\left(100 \mathrm{MHz}, \mathrm{CDCl}_{3}\right)$ $\delta$ 170.4, 134.8, 121.8, 84.7, 81.8, 67.5, 21.4, 20.5, 18.2, 4.3; FTIR ( $v_{\max }$ ) $\mathrm{cm}^{-1}$ ) 2980, 2918, 2228, 1731, 1638, 1443, 1370, 1234, 1154, 1040, 1015, 943, 864, 610; MS (EI, $m / z) 166\left(13, \mathrm{M}^{+}\right), 151(30), 123$ (100), 109 (79), 91 (87), 79 (26).<smiles>CC#CC(C)=CC(C)OC(C)=O</smiles>

(E)-1.2a. ${ }^{1} \mathrm{H}$ NMR (400 MHz, $\left.\mathrm{CDCl}_{3}\right) \delta 5.66(\mathrm{~d}, J=9.2 \mathrm{~Hz}, 1 \mathrm{H})$, $5.43(\mathrm{dq}, J=9.2,6.4 \mathrm{~Hz}, 1 \mathrm{H}), 3.75(\mathrm{~s}, 3 \mathrm{H}), 1.92(\mathrm{~s}, 3 \mathrm{H}), 1.87$ (d, $J=$ $1.2 \mathrm{~Hz}, 3 \mathrm{H}), 1.33(\mathrm{~d}, J=6.8 \mathrm{~Hz}, 3 \mathrm{H}) ;{ }^{13} \mathrm{C} \mathrm{NMR}\left(100 \mathrm{MHz}, \mathrm{CDCl}_{3}\right) \delta$ 155.3, 134.0, 122.4, 85.0, 81.7, 71.6, 54.7, 20.5, 18.2, 4.3; FTIR ( $v_{\max }$ ) $\mathrm{cm}^{-1}$ ) 2981, 2957, 2920, 2853, 2227, 1742, 1639, 1441, 1327, 1255, 1154, 1034, 938, 898, 864, 791; MS (EI, $m / z) 182\left(13, \mathrm{M}^{+}\right), 167$ (5), 123 (100), 107 (84), 91 (99), 79 (62).<smiles>CC#CC(C)=CC(C)OC(C)C</smiles>

(E)-1.2b. ${ }^{1} \mathrm{H}$ NMR (400 MHz, $\left.\mathrm{CDCl}_{3}\right) \delta 5.67(\mathrm{~d}, J=8.8 \mathrm{~Hz} 1 \mathrm{H})$, $5.48(\mathrm{dq}, J=8.8,6.5 \mathrm{~Hz}, 1 \mathrm{H}), 3.75(\mathrm{~s}, 3 \mathrm{H}), 2.29(\mathrm{t}, J=7.0 \mathrm{~Hz}, 2 \mathrm{H}), 1.88$ $(\mathrm{s}, 3 \mathrm{H}), 1.50$ (quint, $J=6.8 \mathrm{~Hz}, 2 \mathrm{H}), 1.41$ (sext, $J=7.6 \mathrm{~Hz}, 2 \mathrm{H}), 1.34$ (d, $J=6.4 \mathrm{~Hz}, 3 \mathrm{H}), 0.92(\mathrm{t}, J=7.2 \mathrm{~Hz}, 3 \mathrm{H}) ;{ }^{13} \mathrm{C} \mathrm{NMR}:\left(101 \mathrm{MHz}, \mathrm{CDCl}_{3}\right)$ $\delta 155.3,133.9,122.5,89.6,82.6,71.7,54.7,30.9,22.1,20.5,19.1,18.3$, 13.7; FTIR $\left(v_{\max } / \mathrm{cm}^{-1}\right) 2958,2933,2873,2220,1743,1638$, 1441, 1326, 1257, 1154, 1036, 939, 865, 792; MS (EI, $m / z) 224$ (6, $\mathrm{M}^{+}$), 182 (9), 165 (70), 149 (50), 133 (19), 119 (41), 105 (77), 91 (100), 77 (53).<smiles>COC(C)C=C(C)C#CBr</smiles>

(E)-1.2f. ${ }^{1} \mathrm{H}$ NMR (400 MHz, $\left.\mathrm{CDCl}_{3}\right) \delta 5.62(\mathrm{~d}, J=9.2,1 \mathrm{H})$, $5.26-5.32(\mathrm{~m}, 1 \mathrm{H}), 3.75(\mathrm{~s}, 3 \mathrm{H}), 1.93(\mathrm{~s}, 1 \mathrm{H}), 1.88(\mathrm{~d}, J=1.2 \mathrm{~Hz}, 3 \mathrm{H})$, $1.68-1.77(\mathrm{~m}, 1 \mathrm{H}), 1.49-1.58(\mathrm{~s}, 1 \mathrm{H}), 1.37-1.24(\mathrm{~m}, 4 \mathrm{H}), 0.88(\mathrm{t}, J=$ $6.6 \mathrm{~Hz}, 3 \mathrm{H}) ;{ }^{13} \mathrm{C} \mathrm{NMR}\left(101 \mathrm{MHz}, \mathrm{CDCl}_{3}\right) \delta 155.4,133.1,123.2,85.0$, 82.0, 75.2, 55.0, 34.2, 27.1, 23.0, 18.4, 14.1, 4.3; FTIR $\left(v_{\max } / \mathrm{cm}^{-1}\right)$ 2957, 2930, 2862, 2222, 1743, 1638, 1441, 1380, 1321, 1259, 1152, 1091, 1036, 933, 879, 866, 791; MS (EI, $m / z) 224\left(4, \mathrm{M}^{+}\right), 167$ (31), 148 (26), 133 (19), 123 (38), 119 (51), 105 (47), 91 (94), 77 (56).<smiles>CC#CC(C)=CC(Br)OC(C)=O</smiles>

(E)-1.2g. ${ }^{1} \mathrm{H}$ NMR (400 MHz, $\left.\mathrm{CDCl}_{3}\right) \delta 5.63(\mathrm{~d}, J=9.6 \mathrm{~Hz}, 1 \mathrm{H})$, $5.07(\mathrm{dd}, J=9.6,7.0 \mathrm{~Hz}, 1 \mathrm{H}), 3.75(\mathrm{~s}, 3 \mathrm{H}), 1.93(\mathrm{~s}, 3 \mathrm{H}), 1.89(\mathrm{~d}, J=1.2$ $\mathrm{Hz}, 3 \mathrm{H}), 0.95(\mathrm{~d}, J=6.8 \mathrm{~Hz}, 3 \mathrm{H}), 0.90(\mathrm{~d}, J=6.8 \mathrm{~Hz}, 3 \mathrm{H}) ;{ }^{13} \mathrm{C} \mathrm{NMR}$ $\left(100 \mathrm{MHz}, \mathrm{CDCl}_{3}\right) \delta 155.6,131.5,124.0,85.0,81.9,79.6,54.8,32.5$, $18.5,18.3,17.9,4.3 ;$ FTIR $\left(v_{\max } / \mathrm{cm}^{-1}\right) 2960,2920,2876,2222,1743,1639$, 1441, 1254, 966, 934, 791; MS (EI, $m / z) 210\left(9, \mathrm{M}^{+}\right), 195$ (3), 167 (100), 151 (85), 135 (41), 123 (42), 119 (92), 108 (71), 91 (82), 77 (59).<smiles>CC#CC(C)=CC(CCC)OC(C)C</smiles>

(E)-1.2h. ${ }^{1} \mathrm{H}$ NMR (400 MHz, $\left.\mathrm{CDCl}_{3}\right) \delta 7.41-7.45(\mathrm{~m}, 2 \mathrm{H})$, $7.28-7.33(\mathrm{~m}, 3 \mathrm{H}), 5.87(\mathrm{dq}, J=8.8,1.2 \mathrm{~Hz}, 1 \mathrm{H}), 5.50(\mathrm{dq}, J=8.8,6.4$ $\mathrm{Hz}, 1 \mathrm{H}), 3.77(\mathrm{~s}, 3 \mathrm{H}), 2.00(\mathrm{~d}, J=1.2 \mathrm{~Hz}, 3 \mathrm{H}), 1.39(\mathrm{~d}, J=6.4 \mathrm{~Hz}, 3 \mathrm{H})$; ${ }^{13} \mathrm{C} \mathrm{NMR}\left(101 \mathrm{MHz}, \mathrm{CDCl}_{3}\right) \delta 155.3,135.5,131.7,128.4,123.3,121.9$, 91.3, 88.5, 71.6, 54.8, 20.4, 18.0; FTIR $\left(\nu_{\max } / \mathrm{cm}^{-1}\right)$ 3059, 2982, 2956, 
2923, 1741, 1489, 1441, 1328, 1255, 1142, 1036, 942, 866, 754, 690; MS $($ EI, $m / z) 244\left(6, \mathrm{M}^{+}\right), 185$ (100), 167 (90), 153 (95), 152 (88), 141 (44), 128 (38), 115 (54), 102 (22), 91 (35), 77 (36).<smiles>COC(C)/C=C(\C)C#Cc1ccccc1</smiles>

(E)-1.2n. ${ }^{1} \mathrm{H}$ NMR (400 MHz, $\left.\mathrm{C}_{6} \mathrm{D}_{6}\right) \delta 5.99\left(\mathrm{dd}, J_{\mathrm{AB}}=15.6,6.8 \mathrm{~Hz}\right.$, $1 \mathrm{H}), 5.67\left(\mathrm{~d}, J_{\mathrm{AB}}=15.6 \mathrm{~Hz}, 1 \mathrm{H}\right), 5.15$ (quint, $\left.J=6.6 \mathrm{~Hz}, 1 \mathrm{H}\right), 3.73(\mathrm{~s}$, $3 \mathrm{H}), 1.90(\mathrm{~d}, J=1.6 \mathrm{~Hz}, 3 \mathrm{H}), 1.33(\mathrm{~d}, J=6.8 \mathrm{~Hz}, 3 \mathrm{H}) ;{ }^{13} \mathrm{C}$ NMR $(100$ $\left.\mathrm{MHz}, \mathrm{C}_{6} \mathrm{D}_{6}\right) \delta 155.0,139.6,112.9,87.9,75.4,54.7,20.1,4.3$; FTIR $\left(v_{\max } / \mathrm{cm}^{-1}\right)$ 2984, 2958, 2920, 2854, 2223, 1743, 1638, 1441, 1255, 1036, 941, 871, 790; MS (EI, $m / z) 168\left(3, \mathrm{M}^{+}\right), 153$ (3), 109 (100), 91 (83), 77 (47).<smiles>CC#CC=CC(C)OC</smiles>

General Method for the Rhodium-Catalyzed Synthesis of Vinylallenes with (Z)-2-En-4-yne Acetates and Arylboronic Acids. To an oven-dried flask containing $\mathbf{1 . 1}(0.3 \mathrm{mmol})$ and $2(0.9$ mmol, 3 equiv) was added $\mathrm{CsF}\left(0.45 \mathrm{mmol}, 1.5\right.$ equiv), $[\mathrm{RhOH}(\mathrm{cod})]_{2}$ $(6 \mathrm{~mol} \% \mathrm{Rh})$, degassed water $(0.1 \mathrm{~mL})$, and dry THF $(2.0 \mathrm{~mL})$, successively, under Ar. The reaction mixture was stirred magnetically at room temperature for $16 \mathrm{~h}$. The solvent was removed under reduced pressure, and the residue was purified by column chromatography using hexane/ethyl acetate as eluent to give 3 .

General Method for the Palladium-Catalyzed Synthesis of Vinylallenes with (Z)-or (E)-2-En-4-yne Carbonates and Arylboronic Acids. A mixture of $\mathrm{Pd}_{2}(\mathrm{dba})_{3} \mathrm{CHCl}_{3}(3 \% \mathrm{Pd})$ and $\mathrm{PPh}_{3}$ $(12 \%)$ in dry THF $(1 \mathrm{~mL})$ was stirred for $15 \mathrm{~min}$ under Ar. Then, the dry THF ( $4 \mathrm{~mL})$ solution of $\mathbf{1 . 2}(0.2 \mathrm{mmol}), \mathbf{2}(0.6 \mathrm{mmol}, 3$ equiv), and degassed water $(0.4 \mathrm{~mL})$ was added successively. The mixture was stirred magnetically in an oil bath preheated at $65^{\circ} \mathrm{C}$. The solvent was evaporated, and the residue was purified by column chromatography on silica gel (hexane/ethyl acetate), affording the product 3 .

Computational Details. The geometry optimizations were performed by using the density functional theory (DFT) with Becke three parameter Lee, Yang, and Parr, (B3LYP $)^{28}$ functional and LANL2DZ basis set implemented in Gaussian 09 software. ${ }^{29}$ The imaginary frequencies have been checked with the frequencies for these structures. The geometry analysis is shown in Supporting Information for some selected geometrical parameters. The natural Bond Orbital (NBO) and Mulliken charges were used for the charge analysis.

3aa. ${ }^{1} \mathrm{H}$ NMR $\left(400 \mathrm{MHz}, \mathrm{CDCl}_{3}\right) \delta 7.34-7.38(\mathrm{~m}, 2 \mathrm{H}), 7.28-7.33$ $(\mathrm{m}, 2 \mathrm{H}), 7.14-7.21(\mathrm{~m}, 1 \mathrm{H}), 6.06(\mathrm{dq}, J=15.6,1.6 \mathrm{~Hz}, 1 \mathrm{H}), 5.64(\mathrm{dq}$, $J=16.0,6.8 \mathrm{~Hz}, 1 \mathrm{H}), 2.09(\mathrm{~s}, 3 \mathrm{H}), 1.90(\mathrm{~s}, 3 \mathrm{H}), 1.80(\mathrm{dd}, J=6.8,1.6 \mathrm{~Hz}$, $3 \mathrm{H}) ;{ }^{1} \mathrm{H}$ NMR $\left(400 \mathrm{MHz}, \mathrm{C}_{6} \mathrm{D}_{6}\right) \delta 7.40-7.46(\mathrm{~m}, 2 \mathrm{H}), 7.14-7.21(\mathrm{~m}$, $2 \mathrm{H}), 7.02-7.08(\mathrm{~m}, 1 \mathrm{H}), 6.15(\mathrm{dq}, J=15.6,1.6 \mathrm{~Hz}, 1 \mathrm{H}), 5.51(\mathrm{dq}, J=$ 15.6, $6.4 \mathrm{~Hz}, 1 \mathrm{H}), 2.02$ (s, 3H), 1.85 (s, 3H), 1.63 (dd, $J=6.8,1.2 \mathrm{~Hz}$, $3 \mathrm{H}) ;{ }^{13} \mathrm{C} \mathrm{NMR}\left(100 \mathrm{MHz}, \mathrm{CDCl}_{3}\right) \delta 207.0,137.8,129.2,128.3,126.4$, $125.8,124.5,101.8,99.6,18.3,17.1,15.5 ;{ }^{13} \mathrm{CNMR}\left(101 \mathrm{MHz}, \mathrm{C}_{6} \mathrm{D}_{6}\right) \delta$ 207.5, 138.2, 129.9, 128.7, 126.9, 126.4, 124.5, 102.3, 100.4, 18.3, 17.3, 15.6; FTIR $\left(v_{\max } / \mathrm{cm}^{-1}\right) 3029,2981,2911,1933,1598,1491,1444$, $1367,1065,1026,961,757,690,603,594$; MS (EI, $m / z) 184\left(100, \mathrm{M}^{+}\right)$, 169 (98), 154 (86), 141 (70), 128 (51), 115 (36), 105 (19), 91 (30), 77 (15); HRMS (ESI) calcd for $\mathrm{C}_{14} \mathrm{H}_{17}\left(\mathrm{MH}^{+}\right) 185.1325$, found 185.1324 .

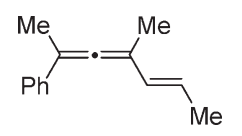

3ab. ${ }^{1} \mathrm{H}$ NMR (400 MHz, $\left.\mathrm{CDCl}_{3}\right) \delta 7.54\left(\mathrm{~d}, J_{\mathrm{AB}}=8.0 \mathrm{~Hz}, 2 \mathrm{H}\right), 7.44$ $\left(\mathrm{d}, J_{\mathrm{AB}}=8.0 \mathrm{~Hz}, 2 \mathrm{H}\right), 6.05(\mathrm{dq}, J=16.0,2.0 \mathrm{~Hz}, 1 \mathrm{H}), 5.64(\mathrm{dq}, J=15.6,6.8$
$\mathrm{Hz}, 1 \mathrm{H}), 2.1$ (s, 3H), 1.91 (s, 3H), 1.79 (dd, $J=6.8,1.6 \mathrm{~Hz}, 3 \mathrm{H}) ;{ }^{1} \mathrm{H}$ NMR $\left(400 \mathrm{MHz}, \mathrm{C}_{6} \mathrm{D}_{6}\right) \delta 7.35(\mathrm{~d}, J=8.4 \mathrm{~Hz}, 2 \mathrm{H}), 7.20(\mathrm{~d}, J=8.4 \mathrm{~Hz}, 2 \mathrm{H}), 6.08$ (dd, $J=15.2,1.6 \mathrm{~Hz}, 1 \mathrm{H}), 5.50(\mathrm{dq}, J=15.6,6.4 \mathrm{~Hz}, 1 \mathrm{H}), 1.87(\mathrm{~s}, 3 \mathrm{H})$, $1.80(\mathrm{~s}, 3 \mathrm{H}), 1.62(\mathrm{dd}, J=6.8,1.6 \mathrm{~Hz}, 3 \mathrm{H}) ;{ }^{13} \mathrm{C} \mathrm{NMR}\left(100 \mathrm{MHz}, \mathrm{CDCl}_{3}\right)$ $\delta 207.8,141.7,128.5,128.3(\mathrm{q}, J=32 \mathrm{~Hz}), 125.9,125.4,125.1(\mathrm{q}, J=3.9$ $\mathrm{Hz}), 124.4(\mathrm{q}, J=270 \mathrm{~Hz}), 102.5,99.0,18.3,17.0,15.3 ;{ }^{13} \mathrm{C}$ NMR $(100$ $\left.\mathrm{MHz}, \mathrm{C}_{6} \mathrm{D}_{6}\right) \delta 208.2,141.9,128.9(\mathrm{q}, J=32 \mathrm{~Hz}), 128.9,126.4,125.5$ (q, $J$ $=3.9 \mathrm{~Hz}), 125.0(\mathrm{q}, J=263 \mathrm{~Hz}), 102.9,99.6,18.4,17.0,15.4$; FTIR $\left(v_{\max } /\right.$ $\mathrm{cm}^{-1}$ ) 2987, 2928, 2855, 1933, 1616, 1323, 1164, 1114, 1074, 1014, 840, 607; MS (EI, $m / z) 252\left(100, \mathrm{M}^{+}\right), 237$ (82), 222 (21), 209 (43), 197 (64), 183 (26), 168 (36), 153 (39), 141 (17), 128 (14), 115 (13), 91 (16); HRMS (EI) calcd for $\mathrm{C}_{15} \mathrm{H}_{15} \mathrm{~F}_{3}\left(\mathrm{M}^{+}\right)$252.1120, found 252.111 .<smiles>CC=CC(C)=Cc1ccc(C(F)(F)F)cc1</smiles>

3ac. ${ }^{1} \mathrm{H} \mathrm{NMR}\left(400 \mathrm{MHz}, \mathrm{CDCl}_{3}\right) \delta 7.89(\mathrm{~d}, J=8.4 \mathrm{~Hz}, 2 \mathrm{H}), 7.43(\mathrm{~d}$, $\left.J_{\mathrm{AB}}=8.0 \mathrm{~Hz}, 2 \mathrm{H}\right), 6.04\left(\mathrm{dq}, J_{\mathrm{AB}}=15.6,1.6 \mathrm{~Hz}, 1 \mathrm{H}\right), 5.68(\mathrm{dq}, J=15.2$, $6.8 \mathrm{~Hz}, 1 \mathrm{H}), 2.57$ (s, 3H), 2.1 (s, 3H), 1.9 (s, 3H), 1.80 (dd, $J=6.4,1.2$ $\mathrm{Hz}, 3 \mathrm{H}) ;{ }^{13} \mathrm{C}$ NMR $\left(100 \mathrm{MHz}, \mathrm{CDCl}_{3}\right) \delta 208.3,197.7,143.0,135.0$, 128.43, 128.39, 125.8, 125.4, 102.4, 99.4, 26.6, 18.4, 17.0, 15.3; FTIR $\left(v_{\max } / \mathrm{cm}^{-1}\right) 2925,2850,1930,1681,1601,1357,1266,959,838,646$, 602; MS (EI, $m / z) 226\left(100, \mathrm{M}^{+}\right), 211$ (57), 183 (37), 168 (43), 153 (39), 141 (24), 128 (19), 115 (21), 91 (11); HRMS (ESI) calcd for $\mathrm{C}_{16} \mathrm{H}_{19}\left(\mathrm{MH}^{+}\right)$227.1430, found 227.1429.<smiles>C/C=C/C(C)=C(C)c1ccc(OC)cc1</smiles>

3ad. ${ }^{1} \mathrm{H}$ NMR $\left(400 \mathrm{MHz}, \mathrm{CDCl}_{3}\right) \delta 7.24\left(\mathrm{~d}, J_{\mathrm{AB}}=8.0,2 \mathrm{H}\right), 7.11(\mathrm{~d}$, $\left.J_{\mathrm{AB}}=8.0 \mathrm{~Hz}, 2 \mathrm{H}\right), 6.06(\mathrm{dq}, J=16.0,2.0 \mathrm{~Hz}, 1 \mathrm{H}), 5.64(\mathrm{dq}, J=15.6,6.4$ $\mathrm{Hz}, 1 \mathrm{H}), 2.32$ (s, 3H), 2.07 (s, 3H), $1.80(\mathrm{~s}, 3 \mathrm{H}), 1.79$ (dd, $J=6.8,1.6$ $\mathrm{Hz}, 3 \mathrm{H}) ;{ }^{13} \mathrm{C} \mathrm{NMR}\left(100 \mathrm{MHz}, \mathrm{CDCl}_{3}\right) \delta 206.7,136.1,134.8,129.5$, 129.0, 125.7, 124.3, 101.7, 99.5, 21.1, 18.3, 17.2, 15.5; FTIR ( $v_{\max }$ ) $\left.\mathrm{cm}^{-1}\right)$ 3038; 2982, 2921, 2850, 1510, 1444, 1370, 1025, 963, 816, 590; MS (EI, $m / z) 198\left(100, \mathrm{M}^{+}\right), 183$ (93), $168(82), 153$ (48), 141 (36), 128 (33), 115 (35), 105 (18), 91 (30), 77 (19); HRMS (ESI) calcd for $\mathrm{C}_{15} \mathrm{H}_{19}\left(\mathrm{MH}^{+}\right)$199.1481, found 199.1481.<smiles>CC=CC(C)=C=C(C)c1ccc(C)cc1</smiles>

3ae. ${ }^{1} \mathrm{H}$ NMR $\left(400 \mathrm{MHz}, \mathrm{CDCl}_{3}\right.$ ) $\delta 7.20-7.41(\mathrm{~m}, 3 \mathrm{H}), 7.02-7.05$ $(\mathrm{m}, 1 \mathrm{H}), 6.10(\mathrm{dq}, J=15.2,1.6 \mathrm{~Hz}, 1 \mathrm{H}), 5.67(\mathrm{dq}, J=15.2,6.4 \mathrm{~Hz}, 1 \mathrm{H})$, 2.37 (s, 3H), 2.11 (s, 3H), $1.93(\mathrm{~s}, 3 \mathrm{H}), 1.83$ (dd, J = 6.8, $1.6 \mathrm{~Hz}, 3 \mathrm{H})$; ${ }^{1} \mathrm{H}$ NMR (400 MHz, $\left.\mathrm{C}_{6} \mathrm{D}_{6}\right) \delta 7.35(\mathrm{~s}, 1 \mathrm{H}), 7.27-7.33(\mathrm{~m}, 1 \mathrm{H})$, $7.12-7.16(\mathrm{~m}, 1 \mathrm{H}), 6.88-6.94(\mathrm{~m}, 1 \mathrm{H}), 6.19(\mathrm{dq}, J=15.6,1.6 \mathrm{~Hz}, 1 \mathrm{H})$, $5.50(\mathrm{dq}, J=15.2,6.4 \mathrm{~Hz}, 1 \mathrm{H}), 2.12(\mathrm{~s}, 3 \mathrm{H}), 2.06(\mathrm{~s}, 3 \mathrm{H}), 1.87$ $(\mathrm{s}, 3 \mathrm{H}), 1.62(\mathrm{dd}, J=6.8,1.6 \mathrm{~Hz}, 3 \mathrm{H}) ;{ }^{13} \mathrm{C} \mathrm{NMR}\left(100 \mathrm{MHz}, \mathrm{CDCl}_{3}\right) \delta$ 207.0, 138.79, 137.8, 129.4, 128.2, 127.3, 126.6, 124.3, 123.0, 101.6, 99.6, 21.6, 18.3, 17.3, 15.5; ${ }^{13} \mathrm{C}$ NMR $\left(100 \mathrm{MHz}, \mathrm{C}_{6} \mathrm{D}_{6}\right) \delta 207.5$, $138.2,138.0,130.0,128.7,127.8,127.1,124.5,123.6,102.2,100.4$, $21.5,18.4,17.5,15.7$; FTIR $\left(v_{\max } / \mathrm{cm}^{-1}\right) 3013,2918,2855,1933$, 1604, 1488, 1444, 1376, 1028, 962, 781, 696; MS (EI, $m / z) 198$ (100, $\mathrm{M}^{+}$), 183 (87), 168 (78), 153 (47), 141 (37), 128 (32), 115 (34), 105 (17), 91 (32); HRMS (ESI) calcd for $\mathrm{C}_{15} \mathrm{H}_{19}\left(\mathrm{MH}^{+}\right)$199.1481, found 199.1481. 
<smiles>C/C=C/C(C)=C(/C)c1cccc(C)c1</smiles>

3af. ${ }^{1} \mathrm{H}$ NMR $\left(400 \mathrm{MHz}, \mathrm{CDCl}_{3}\right) \delta 7.31-7.32(\mathrm{~m}, 1 \mathrm{H}), 7.21-7.23$ $(\mathrm{m}, 2 \mathrm{H}), 7.13-7.17(\mathrm{~m}, 1 \mathrm{H}), 6.04(\mathrm{dq}, J=15.6,1.6 \mathrm{~Hz}, 1 \mathrm{H}), 5.67(\mathrm{dq}$, $J=15.6,6.8 \mathrm{~Hz}, 1 \mathrm{H}), 2.07(\mathrm{~s}, 3 \mathrm{H}), 1.89(\mathrm{~s}, 3 \mathrm{H}), 1.81(\mathrm{dd}, J=6.8,1.6 \mathrm{~Hz}$, $3 \mathrm{H}) ;{ }^{13} \mathrm{C} \mathrm{NMR}\left(100 \mathrm{MHz}, \mathrm{CDCl}_{3}\right) \delta 207.3,140.1,134.4,129.5,128.9$, $126.5,126.0,125.3,124.1,102.5,99.0,18.5,17.3,15.6$; FTIR ( $v_{\max } /$ $\mathrm{cm}^{-1}$ ) 3034, 2982, 2916, 2855, 1933, 1592, 1566, 1475, 1443, 1417, $1368,1106,1080,1067,1027,961,776,748,687,609$; MS (EI, $m / z)$ 218 (97, M ${ }^{+}$), 203 (70), 183 (33), 175 (19), 168 (100), 153 (77), 141 (45), 128 (31), 115 (33), 102 (12), 91 (21), 77 (20); HRMS (ESI) calcd for $\mathrm{C}_{14} \mathrm{H}_{16} \mathrm{Cl}\left(\mathrm{MH}^{+}\right)$219.0935, found 219.0936 .<smiles>C/C=C/C(C)=C(/C)c1cccc(Cl)c1</smiles>

3ag. ${ }^{1} \mathrm{H} \mathrm{NMR}\left(400 \mathrm{MHz}, \mathrm{CDCl}_{3}\right) \delta 7.20-7.41(\mathrm{~m}, 2 \mathrm{H}), 6.84-6.93$ $(\mathrm{m}, 2 \mathrm{H}), 6.10(\mathrm{dq}, J=15.6,1.6 \mathrm{~Hz}, 1 \mathrm{H}), 5.54(\mathrm{dq}, J=16.0,6.8 \mathrm{~Hz}, 1 \mathrm{H})$, $3.81(\mathrm{~s}, 3 \mathrm{H}), 2.07(\mathrm{~s}, 3 \mathrm{H}), 1.84(\mathrm{~s}, 3 \mathrm{H}), 1.78(\mathrm{dd}, J=6.4,1.6 \mathrm{~Hz}, 3 \mathrm{H}) ;{ }^{1} \mathrm{H}$ $\operatorname{NMR}\left(400 \mathrm{MHz}, \mathrm{C}_{6} \mathrm{D}_{6}\right) \delta 7.36(\mathrm{dd}, J=7.6,1.6 \mathrm{~Hz}, 1 \mathrm{H}), 7.06(\mathrm{dt}, J=8.0$, $1.2 \mathrm{~Hz}, 1 \mathrm{H}), 6.86(\mathrm{t}, J=7.6 \mathrm{~Hz}, 1 \mathrm{H}), 6.56(\mathrm{~d}, J=8.4 \mathrm{~Hz}, 1 \mathrm{H}), 6.28(\mathrm{dq}, J$ $=15.6,1.6 \mathrm{~Hz}, 1 \mathrm{H}), 5.46(\mathrm{dq}, J=15.6,6.4 \mathrm{~Hz}, 1 \mathrm{H}), 3.33(\mathrm{~s}, 3 \mathrm{H}), 2.22(\mathrm{~s}$, $3 \mathrm{H}), 1.88(\mathrm{~s}, 3 \mathrm{H}), 1.64(\mathrm{dd}, J=6.4,1.6 \mathrm{~Hz}, 3 \mathrm{H}) ;{ }^{13} \mathrm{C} \mathrm{NMR}(100 \mathrm{MHz}$, $\left.\mathrm{CDCl}_{3}\right) \delta 207.5,157.0,130.1,129.2,128.1,127.9,123.4,120.5,111.4$, 98.5, 97.2, 55.7, 19.6, 18.3, 15.7; ${ }^{13} \mathrm{C}$ NMR $\left(101 \mathrm{MHz}, \mathrm{C}_{6} \mathrm{D}_{6}\right) \delta 208.2$, 157.6, 130.9, 129.9, 128.8, 127.9, 123.3, 120.9, 111.7, 99.1, 98.3, 55.2, 20.0, 18.4, 16.0; FTIR $\left(v_{\max } / \mathrm{cm}^{-1}\right) 2924,1596,1578,1491,1434,1245$, 1029, 962, 749, 603; MS (EI, $m / z) 214$ (23, M+), 199 (100), 184 (38), 165 (34), 152 (25), 141 (25), 135 (78), 128 (29), 115 (34), 91 (30), 77 (28); HRMS (ESI) calcd for $\mathrm{C}_{15} \mathrm{H}_{19}\left(\mathrm{MH}^{+}\right) 215.1430$, found 215.1430 .

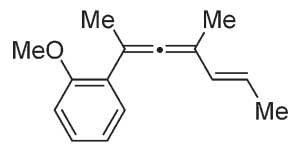

3ah. ${ }^{1} \mathrm{H}$ NMR (500 MHz, $\left.\mathrm{CDCl}_{3}\right) \delta 7.78-7.87(\mathrm{~m}, 2 \mathrm{H}), 7.70-7.77$ $(\mathrm{m}, 2 \mathrm{H}), 7.54-7.59(\mathrm{~m}, 1 \mathrm{H}), 7.40-7.52(\mathrm{~m}, 2 \mathrm{H}), 6.14(\mathrm{dq}, J=16.0,1.0$ $\mathrm{Hz}, 1 \mathrm{H}), 5.72(\mathrm{dq}, J=15.5,6.5 \mathrm{~Hz}, 1 \mathrm{H}), 2.25(\mathrm{~s}, 3 \mathrm{H}), 1.97(\mathrm{~s}, 3 \mathrm{H}), 1.85$ $(\mathrm{d}, J=5.5 \mathrm{~Hz}, 3 \mathrm{H}) ;{ }^{1} \mathrm{H}$ NMR $\left(400 \mathrm{MHz}, \mathrm{C}_{6} \mathrm{D}_{6}\right) \delta 7.72-7.77(\mathrm{~m}, 2 \mathrm{H})$, $7.58-7.67(\mathrm{~m}, 3 \mathrm{H}), 7.20-7.30(\mathrm{~m}, 2 \mathrm{H}), 6.19(\mathrm{dq}, J=15.6,2.4 \mathrm{~Hz}, 1 \mathrm{H})$, $5.54(\mathrm{dq}, J=15.6,6.8 \mathrm{~Hz}, 1 \mathrm{H}), 2.14(\mathrm{~s}, 3 \mathrm{H}), 1.89(\mathrm{~s}, 3 \mathrm{H}), 1.64(\mathrm{dd}, J=6.4$, $1.6 \mathrm{~Hz}, 3 \mathrm{H}) ;{ }^{13} \mathrm{C} \mathrm{NMR}\left(126 \mathrm{MHz}, \mathrm{CDCl}_{3}\right) \delta 208.0,135.4,133.8,132.5$, 129.3, 128.1, 127.71, 127.68, 126.2, 125.6, 125.5, 124.8, 123.5, 102.2, $100.1,18.5,17.4,15.7 ;{ }^{13} \mathrm{C} \mathrm{NMR}\left(101 \mathrm{MHz}, \mathrm{C}_{6} \mathrm{D}_{6}\right) \delta 208.3,135.6,134.4$, 133.0, 129.7, 128.4, 128.2, 126.4, 125.9, 125.8, 124.8, 124.0, 102.6, 100.7, 18.4, 17.4, 15.7; FTIR $\left(v_{\max } / \mathrm{cm}^{-1}\right)$ 3053, 2982, 2915, 1929, 1629, 1597, 1505, 1371, 1025, 961, 854, 817, 783, 745; MS (EI, $m / z) 234\left(100, \mathrm{M}^{+}\right)$, 219 (88), 204 (81), 189 (27), 178 (43), 165 (25), 152 (20), 141 (15); HRMS (ESI) calcd for $\mathrm{C}_{18} \mathrm{H}_{19}\left(\mathrm{MH}^{+}\right)$235.1481, found 235.1481.

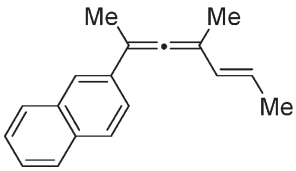

3ai. ${ }^{1} \mathrm{H}$ NMR (400 MHz, $\mathrm{CDCl}_{3}$ ) $\delta 7.20-7.23(\mathrm{~m}, 1 \mathrm{H}), 7.01-7.05$ $(\mathrm{m}, 2 \mathrm{H}), 6.27(\mathrm{dq}, J=15.6,1.6 \mathrm{~Hz}, 1 \mathrm{H}), 5.96(\mathrm{dq}, J=15.6,6.4 \mathrm{~Hz}, 1 \mathrm{H})$, $2.06(\mathrm{~s}, 3 \mathrm{H}), 1.87(\mathrm{~s}, 3 \mathrm{H}), 1.79(\mathrm{dd}, J=6.8,1.2 \mathrm{~Hz}, 3 \mathrm{H}) ;{ }^{13} \mathrm{C}$ NMR $(100$ $\left.\mathrm{MHz}, \mathrm{CDCl}_{3}\right) \delta 206.8,140.1,129.3,126.9,125.2,124.5,118.7,103.3$, 99.1, 18.3, 17.7, 15.6; FTIR $\left(v_{\max } / \mathrm{cm}^{-1}\right) 3103,2987,2922,2855,1929$, $1442,1368,1247,1183,1026,962,864,775,644 ;$ MS (EI, $m / z) 190$ (95, $\mathrm{M}^{+}$), 175 (100), 160 (45), 147 (32), 142 (59), 134 (23), 129 (17), 115 (25); HRMS (ESI) calcd for $\mathrm{C}_{12} \mathrm{H}_{15} \mathrm{~S}\left(\mathrm{MH}^{+}\right)$191.0889, found 191.0889 .

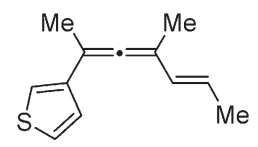

3aj. ${ }^{1} \mathrm{H}$ NMR (400 MHz, $\left.\mathrm{C}_{6} \mathrm{D}_{6}\right) \delta 7.06-7.14(\mathrm{~m}, 1 \mathrm{H}), 6.77-6.84(\mathrm{~m}$, $1 \mathrm{H}), 6.66-6.75(\mathrm{~m}, 1 \mathrm{H}), 6.04(\mathrm{dq}, J=15.6,1.6 \mathrm{~Hz}, 1 \mathrm{H}), 5.48(\mathrm{dq}, J=15.6$, $6.4 \mathrm{~Hz}, 1 \mathrm{H}), 1.80(\mathrm{~s}, 3 \mathrm{H}), 1.76(\mathrm{~s}, 3 \mathrm{H}), 1.61(\mathrm{dd}, J=6.8,1.6 \mathrm{~Hz}, 3 \mathrm{H}) ;{ }^{13} \mathrm{C}$ $\operatorname{NMR}\left(101 \mathrm{MHz}, \mathrm{C}_{6} \mathrm{D}_{6}\right) \delta 207.0,150.8(\mathrm{dd}, J=236,12.2 \mathrm{~Hz}$ ), 149.6 (dd, $J$ $=246,12.9 \mathrm{~Hz}), 135.6(\mathrm{t}, J=5.0 \mathrm{~Hz}), 129.1,125.4,122.1(\mathrm{dd}, J=6.1,3.1$ $\mathrm{Hz}), 117.2(\mathrm{~d}, J=16.8 \mathrm{~Hz}), 115.0(\mathrm{~d}, J=17.5 \mathrm{~Hz}), 18.1,17.0,15.2$; FTIR $\left(v_{\max } / \mathrm{cm}^{-1}\right) 3037,2986,2928,2859,1933,1735,1603,1515,1445,1289$, 962, 817, 773; MS (EI, $m / z) 220\left(100, \mathrm{M}^{+}\right), 205$ (82), 190 (56), 177 (55), 165 (43), 151 (33), 141 (29), 127 (21), 91 (22), 77 (6); HRMS (ESI) calcd for $\mathrm{C}_{14} \mathrm{H}_{15} \mathrm{~F}_{2}\left(\mathrm{MH}^{+}\right)$221.1136, found 221.1137.<smiles>CC=CC(C)=Cc1ccc(F)c(F)c1</smiles>

3ak. ${ }^{1} \mathrm{H}$ NMR $\left(400 \mathrm{MHz}, \mathrm{C}_{6} \mathrm{D}_{6}\right) \delta 8.88-8.92(\mathrm{~m}, 1 \mathrm{H}), 8.42-8.47$ (m, 1H), 7.34-7.37 (m, 1H), 6.70-7.38 (m, 1H), $6.04(\mathrm{dq}, J=15.6,1.2$ $\mathrm{Hz}, 1 \mathrm{H}), 5.48$ (dq, $J=15.6,6.8 \mathrm{~Hz}, 1 \mathrm{H}), 1.86(\mathrm{~s}, 3 \mathrm{H}), 1.76(\mathrm{~s}, 3 \mathrm{H}), 1.6$ (dd, $J=7.2,1.2 \mathrm{~Hz}, 3 \mathrm{H}) ;{ }^{13} \mathrm{C}$ NMR $\left(101 \mathrm{MHz}, \mathrm{C}_{6} \mathrm{D}_{6}\right) \delta 207.4,148.3$, 133.5, 132.6, 129.2, 125.3, 123.1, 103.0, 98.0, 18.3, 16.8, 15.4; FTIR $\left(v_{\max } / \mathrm{cm}^{-1}\right) 3037,2985,2924,2858,1932,1569,1479,1445,1417$, 1378, 1020, 962, 806, 708, 605; MS (EI, $m / z) 185\left(68, \mathrm{M}^{+}\right), 170(100)$, 154 (48), 142 (13), 128 (15), 115 (12), 106 (14), 91 (10), 77 (12); HRMS (EI) calcd for $\mathrm{C}_{13} \mathrm{H}_{15} \mathrm{~N}\left(\mathrm{M}^{+}\right)$185.1199, found 185.1199.<smiles>CC=CC(C)=Cc1cccnc1</smiles>

3al. ${ }^{1} \mathrm{H}$ NMR (500 MHz, $\left.\mathrm{C}_{6} \mathrm{D}_{6}\right) \delta 7.19-7.25(\mathrm{~m}, 1 \mathrm{H}), 6.76-6.85$ $(\mathrm{m}, 3 \mathrm{H}), 6.18(\mathrm{~d}, J=15.5 \mathrm{~Hz}, 1 \mathrm{H}), 5.46(\mathrm{dq}, J=15.5,6.5 \mathrm{~Hz}, 1 \mathrm{H}), 2.10$ $(\mathrm{s}, 3 \mathrm{H}), 1.83(\mathrm{~s}, 3 \mathrm{H}), 1.61(\mathrm{~d}, J=6.5 \mathrm{~Hz}, 3 \mathrm{H}) ;{ }^{13} \mathrm{C} \mathrm{NMR}(126 \mathrm{MHz}$, $\left.\mathrm{C}_{6} \mathrm{D}_{6}\right) \delta 208.7,160.5(\mathrm{~d}, J=248 \mathrm{~Hz}), 129.9,129.6(\mathrm{~d}, J=3.8 \mathrm{~Hz}), 128.5$ $(\mathrm{d}, J=7.6 \mathrm{~Hz}), 128.4,124.5,124.2(\mathrm{~d}, J=3.8 \mathrm{~Hz}), 116 .(\mathrm{d}, J=23.1 \mathrm{~Hz})$, 100.5, 95.8, $19.4(\mathrm{~d}, J=2.0 \mathrm{~Hz}), 18.4,15.7$; FTIR $\left(v_{\max } / \mathrm{cm}^{-1}\right) 3038$, 2923, 2854, 1907, 1576, 1490, 1445, 1368, 1220, 1037, 961, 753, 601; MS (EI, $m / z) 202\left(100, \mathrm{M}^{+}\right), 187$ (74), 165 (57), 159 (52), 152 (50), 146 (44), 133 (35), 123 (35), 109 (30), 91 (14), 77 (9); HRMS (EI) calcd for $\mathrm{C}_{14} \mathrm{H}_{15} \mathrm{~F}\left(\mathrm{M}^{+}\right)$202.1152, found 202.1147.<smiles>C/C=C/C(C)=C\C(C)c1ccccc1F</smiles>

3am. ${ }^{1} \mathrm{H}$ NMR $\left(400 \mathrm{MHz}, \mathrm{C}_{6} \mathrm{D}_{6}\right) \delta 8.40-8.46(\mathrm{~m}, 1 \mathrm{H}), 7.63-7.69$ (m, $1 \mathrm{H}), 7.53-7.58(\mathrm{~m}, 1 \mathrm{H}), 7.33-7.43(\mathrm{~m}, 2 \mathrm{H}), 7.21-7.30(\mathrm{~m}, 2 \mathrm{H})$, $6.26(\mathrm{dq}, J=15.6,1.6 \mathrm{~Hz}, 1 \mathrm{H}), 5.43(\mathrm{dq}, J=15.6,6.8 \mathrm{~Hz}, 1 \mathrm{H}), 2.16(\mathrm{~s}$, $3 \mathrm{H}), 1.83(\mathrm{~s}, 3 \mathrm{H}), 1.62(\mathrm{dd}, J=6.8,1.2 \mathrm{~Hz}, 3 \mathrm{H}) ;{ }^{13} \mathrm{C}$ NMR $(101 \mathrm{MHz}$, $\left.\mathrm{C}_{6} \mathrm{D}_{6}\right) \delta 206.8,138.0,134.7,131.8,130.3,128.9,127.7,126.2,126.1$, 126.0, 125.8, 125.5, 124.2, 99.6, 98.8, 21.7, 18.4, 15.9; FTIR ( $v_{\max }$ ) $\left.\mathrm{cm}^{-1}\right)$ 3057, 2918, 2879, 2723, 1939, 1734, 1439, 1030, 960, 799, 775; MS (EI, $m / z, \mathrm{M}^{+}$): 234 (26), 219 (100), 204 (63), 189 (32), 178 (16), 165 (26), 152 (14); HRMS (EI) calcd for $\mathrm{C}_{18} \mathrm{H}_{18}\left(\mathrm{M}^{+}\right), 234.1403$, found 234.1403 .

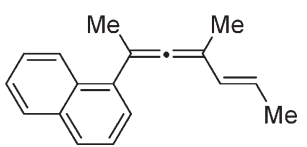


3an. ${ }^{1} \mathrm{H}$ NMR (400 MHz, $\left.\mathrm{C}_{6} \mathrm{D}_{6}\right) \delta 6.08-6.23(\mathrm{~m}, 2 \mathrm{H}), 5.53(\mathrm{dt}, J=$ 15.6, $6.8 \mathrm{~Hz}, 1 \mathrm{H}), 5.45(\mathrm{dq}, J=15.6,6.4 \mathrm{~Hz}, 1 \mathrm{H}), 2.00(\mathrm{q}, J=7.2 \mathrm{~Hz}$, $2 \mathrm{H}), 1.85$ (s, 3H), $1.82(\mathrm{~s}, 3 \mathrm{H}), 1.62$ (dd, $J=6.8,1.6 \mathrm{~Hz}, 3 \mathrm{H}), 1.33$ (sext, $J=7.6 \mathrm{~Hz}, 2 \mathrm{H}), 0.84(\mathrm{t}, J=7.2 \mathrm{~Hz}, 3 \mathrm{H}) ;{ }^{13} \mathrm{C} \mathrm{NMR}\left(101 \mathrm{MHz}, \mathrm{C}_{6} \mathrm{D}_{6}\right) \delta$ 209.5, 130.5, 129.5, 129.2, 123.8, 99.79, 99.75, 35.4, 23.1, 18.3, 16.1, 16.0, 13.9; FTIR $\left(v_{\max } / \mathrm{cm}^{-1}\right)$ 3037, 2959, 2927, 2872, 1929, 1439, 1377, 1032, 961, 586; MS (EI, $\mathrm{m} / z, \mathrm{M}^{+}$): 176 (51), 161 (14), 147 (42), 133 (31), 119 (100), 105 (75), 91 (59), 77 (25); HRMS (EI) calcd for $\mathrm{C}_{13} \mathrm{H}_{20}\left(\mathrm{M}^{+}\right)$176.1560, found 176.1552 .<smiles>C/C=C/C(C)=C(C)/C=C/C(C)C</smiles>

3ba. ${ }^{1} \mathrm{H}$ NMR $\left(400 \mathrm{MHz}, \mathrm{CDCl}_{3}\right) \delta 7.37-7.41(\mathrm{~m}, 2 \mathrm{H}), 7.28-7.34$ $(\mathrm{m}, 2 \mathrm{H}), 7.17-7.23(\mathrm{~m}, 1 \mathrm{H}), 6.10(\mathrm{dq}, J=15.6,1.6 \mathrm{~Hz}, 1 \mathrm{H}), 5.67(\mathrm{dq}$, $J=15.6,6.6 \mathrm{~Hz}, 1 \mathrm{H}), 2.47(\mathrm{t}, J=7.6 \mathrm{~Hz}, 2 \mathrm{H}), 1.93(\mathrm{~s}, 3 \mathrm{H}), 1.83(\mathrm{dd}, J=$ $6.6,2.0 \mathrm{~Hz}, 3 \mathrm{H}$ ), 1.53 (quint, $J=7.2 \mathrm{~Hz}, 2 \mathrm{H}), 1.44$ (sext, $J=7.6 \mathrm{~Hz}, 2 \mathrm{H}$ ), $0.95(\mathrm{t}, J=7.4 \mathrm{~Hz}, 3 \mathrm{H}) ;{ }^{1} \mathrm{H}$ NMR $\left(400 \mathrm{MHz}, \mathrm{C}_{6} \mathrm{D}_{6}\right) \delta 7.45-7.49(\mathrm{~m}$, $2 \mathrm{H}), 7.15-7.22(\mathrm{~m}, 2 \mathrm{H}), 7.03-7.10(\mathrm{~m}, 1 \mathrm{H}), 6.19(\mathrm{dq}, J=15.6,1.6 \mathrm{~Hz}$, $1 \mathrm{H}), 5.67(\mathrm{dq}, J=15.6,6.6 \mathrm{~Hz}, 1 \mathrm{H}), 2.42(\mathrm{t}, J=7.6 \mathrm{~Hz}, 2 \mathrm{H}), 1.87(\mathrm{~s}$, $3 \mathrm{H}), 1.64$ (dd, $J=6.8,1.6 \mathrm{~Hz}, 3 \mathrm{H}), 1.56$ (quint, $J=7.6 \mathrm{~Hz}, 2 \mathrm{H}$ ), 1.36 (sext, $J=7.6 \mathrm{~Hz}, 2 \mathrm{H}), 0.87(\mathrm{t}, J=7.2 \mathrm{~Hz}, 3 \mathrm{H}) ;{ }^{13} \mathrm{C} \mathrm{NMR}(100 \mathrm{MHz}$, $\left.\mathrm{CDCl}_{3}\right) \delta 207.0,137.8,129.6,128.4,126.5,126.3,124.4,105.1,103.2$, $30.3,30.2,22.6,18.5,15.7,14.1 ;{ }^{13} \mathrm{C}$ NMR $\left(101 \mathrm{MHz}, \mathrm{C}_{6} \mathrm{D}_{6}\right) \delta 207.3$, 138.0, 129.9, 128.7, 126.9, 126.7, 124.4, 105.7, 105.6, 30.6, 30.5, 22.9, 18.4, 15.7, 14.2; FTIR $\left(v_{\max } / \mathrm{cm}^{-1}\right)$ 3025, 2957, 2928, 2859, 1931, 1599, 1492, 1446, 1376, 961, 757, 693; MS (EI, $m / z) 226\left(13, \mathrm{M}^{+}\right), 211$ (7), 197 (13), 184 (50), 169 (100), 155 (37), 141 (31), 128 (23), 115 (21), 91 (25), 77 (8); HRMS (EI) calcd for $\mathrm{C}_{17} \mathrm{H}_{22}\left(\mathrm{M}^{+}\right)$226.1716, found 226.1712 .

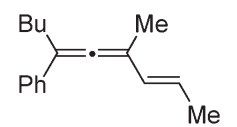

3ca. ${ }^{1} \mathrm{H}$ NMR (400 MHz, $\mathrm{C}_{6} \mathrm{D}_{6}$ ) $\delta 7.44-7.57(\mathrm{~m}, 2 \mathrm{H}), 7.17-7.21$ $(\mathrm{m}, 2 \mathrm{H}), 7.02-7.09(\mathrm{~m}, 1 \mathrm{H}), 6.19(\mathrm{dq}, J=15.6,1.6 \mathrm{~Hz}, 1 \mathrm{H}), 5.54(\mathrm{dq}, J=$ $15.6,6.8 \mathrm{~Hz}, 1 \mathrm{H}), 2.44-2.52(\mathrm{~m}, 1 \mathrm{H}), 1.95-2.03(\mathrm{~m}, 2 \mathrm{H}), 1.87(\mathrm{~s}, 3 \mathrm{H})$, $1.65(\mathrm{dd}, J=6.8,1.6 \mathrm{~Hz}, 3 \mathrm{H}), 1.59-1.73(\mathrm{~m}, 3 \mathrm{H}), 1.24-1.32(\mathrm{~m}, 4 \mathrm{H})$, $1.11-1.19(\mathrm{~m}, 1 \mathrm{H}) ;{ }^{13} \mathrm{C}$ NMR $\left(101 \mathrm{MHz}, \mathrm{C}_{6} \mathrm{D}_{6}\right) \delta 207.1,137.8,130.2$, 128.7, 127.2, 126.8, 124.2, 112.2, 104.3, 38.6, 33.3, 33.5, 27.0, 26.8, 18.4, 15.9; FTIR $\left(v_{\max } / \mathrm{cm}^{-1}\right) 3021,2924,2851,1928,1598,1493,1446,961$, 764, 693; MS (EI, $m / z) 252\left(18, \mathrm{M}^{+}\right), 237$ (18), 223 (31), 195 (26), 183 (100), 169 (50), 155 (48), 141 (30), 128 (29), 115 (25), 91 (36), 77 (12); HRMS (EI) calcd for $\mathrm{C}_{19} \mathrm{H}_{24}\left(\mathrm{M}^{+}\right)$252.1873, found 252.1871.

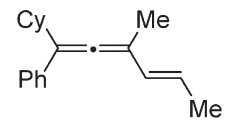

3da. ${ }^{1} \mathrm{H}$ NMR (400 MHz, $\left.\mathrm{C}_{6} \mathrm{D}_{6}\right) \delta$ 7.46-7.53 (m, 2H), 7.16-7.23 $(\mathrm{m}, 2 \mathrm{H}), 7.03-7.09(\mathrm{~m}, 1 \mathrm{H}), 6.14(\mathrm{dd}, J=15.7,1.6 \mathrm{~Hz}, 1 \mathrm{H}), 5.65(\mathrm{dq}, J$ $=15.7,6.7 \mathrm{~Hz}, 1 \mathrm{H}), 2.46(\mathrm{t}, J=8.0 \mathrm{~Hz}, 2 \mathrm{H}), 2.26(\mathrm{t}, J=7.6 \mathrm{~Hz}, 2 \mathrm{H}), 1.66$ (dd, $J=6.8,1.5 \mathrm{~Hz}, 3 \mathrm{H}), 1.54-1.64(\mathrm{~m}, 4 \mathrm{H}), 1.30-1.41(\mathrm{~m}, 4 \mathrm{H}), 0.88$ $(\mathrm{t}, J=7.2 \mathrm{~Hz}, 3 \mathrm{H}), 0.85(\mathrm{t}, J=7.2 \mathrm{~Hz}, 3 \mathrm{H}) ;{ }^{13} \mathrm{C} \mathrm{NMR}\left(101 \mathrm{MHz}, \mathrm{C}_{6} \mathrm{D}_{6}\right)$ $\delta$ 206.8, 138.0, 129.4, 128.7, 126.9, 126.5, 124.1, 108.7, 107.3, 30.8, 30.61, 30.60, 29.6, 23.2, 23.0, 18.6, 14.3, 14.2; FTIR $\left(v_{\max } / \mathrm{cm}^{-1}\right) 3023$, 2956, 2927, 2858, 1597, 1493, 1448, 1377, 961, 757, 692; MS (EI, $m / z)$ $268\left(2, \mathrm{M}^{+}\right), 239$ (4), 226 (3), 211 (39), 183 (33), 169 (100), 155 (38), 141 (48), 128 (14), 115 (17), 91 (28), 77 (8); HRMS (EI) calcd for $\mathrm{C}_{20} \mathrm{H}_{28}\left(\mathrm{M}^{+}\right)$242.1485, found 242.1485 .

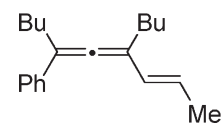

3ea. ${ }^{1} \mathrm{H}$ NMR (400 MHz, $\left.\mathrm{C}_{6} \mathrm{D}_{6}\right) \delta 7.50-7.57$ (m, 4H), $7.12-7.20(\mathrm{~m}, 4 \mathrm{H}), 7.02-7.09(\mathrm{~m}, 2 \mathrm{H}), 6.27(\mathrm{dq}, J=15.6,2.0$ $\mathrm{Hz}, 1 \mathrm{H}), 5.96(\mathrm{dq}, J=15.6,6.8 \mathrm{~Hz}, 1 \mathrm{H}), 2.47(\mathrm{t}, J=7.6 \mathrm{~Hz}, 1 \mathrm{H}), 2.46$ $(\mathrm{t}, J=7.6 \mathrm{~Hz}, 1 \mathrm{H}), 1.62(\mathrm{dd}, J=6.8,1.6 \mathrm{~Hz}, 3 \mathrm{H}), 1.54-1.65(\mathrm{~m}$, $2 \mathrm{H}), 1.31$ (sext, $J=15.6,2 \mathrm{H}), 0.82(\mathrm{t}, J=15.6 \mathrm{~Hz}, 3 \mathrm{H}) ;{ }^{13} \mathrm{C} \mathrm{NMR}$ $\left(101 \mathrm{MHz}, \mathrm{C}_{6} \mathrm{D}_{6}\right) \delta 207.7,137.3,137.1,128.9,128.8,128.7,127.9$, $127.4,127.2$, 127.0, 126.5, 111.3, 109.1, 30.6, 30.5, 23.0, 18.5, 14.2; FTIR $\left(v_{\max } / \mathrm{cm}^{-1}\right) 3026,2956,2929,2858,1597,1492,1445,960$, 756, 692; MS (EI, $m / z) 288\left(8, \mathrm{M}^{+}\right), 259$ (19), 246 (168), 231 (100), 215 (77), 202 (28), 165 (16), 155 (20), 128 (21), 115 (28), 91 (26), 77 (13); HRMS (EI) calcd for $\mathrm{C}_{22} \mathrm{H}_{24}\left(\mathrm{M}^{+}\right)$288.1873, found 288.1876 .

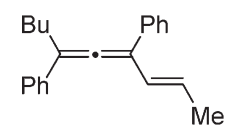

3fa. ${ }^{1} \mathrm{H}$ NMR (400 MHz, $\left.\mathrm{CDCl}_{3}\right) \delta 7.37-7.43(\mathrm{~m}, 2 \mathrm{H})$, $7.31-7.36(\mathrm{~m}, 2 \mathrm{H}), 7.21-7.24(\mathrm{~m}, 1 \mathrm{H}), 6.08(\mathrm{dt}, J=19.6,1.6$ $\mathrm{Hz}, 1 \mathrm{H}), 5.66(\mathrm{dt}, J=15.6,7.2 \mathrm{~Hz}, 1 \mathrm{H}), 2.13-2.20(\mathrm{~m}, 2 \mathrm{H}), 2.13(\mathrm{~s}$, $3 \mathrm{H}), 1.93(\mathrm{~s}, 3 \mathrm{H}), 1.32-1.48(\mathrm{~m}, 4 \mathrm{H}), 0.94(\mathrm{t}, J=7.6 \mathrm{~Hz}, 3 \mathrm{H}) ;{ }^{1} \mathrm{H}$ $\operatorname{NMR}\left(500 \mathrm{MHz}, \mathrm{C}_{6} \mathrm{D}_{6}\right) \delta 7.40-7.46(\mathrm{~m}, 2 \mathrm{H}), 7.12-7.20(\mathrm{~m}, 2 \mathrm{H})$, $7.00-7.09(\mathrm{~m}, 1 \mathrm{H}), 6.20(\mathrm{dt}, J=15.5,1.5 \mathrm{~Hz}, 1 \mathrm{H}), 5.60(\mathrm{dt}, J=12.5$, $6.0 \mathrm{~Hz}, 1 \mathrm{H}), 2.00-2.10(\mathrm{~m}, 2 \mathrm{H}), 2.03(\mathrm{~d}, J=3.0 \mathrm{~Hz}, 3 \mathrm{H}), 1.89(\mathrm{~d}, J=$ 3.0, $3 \mathrm{H}), 1.20-1.22(\mathrm{~m}, 4 \mathrm{H}), 0.84(\mathrm{t}, J=7.5 \mathrm{~Hz}, 3 \mathrm{H}) ;{ }^{13} \mathrm{C} \mathrm{NMR}$ $\left(101 \mathrm{MHz}, \mathrm{CDCl}_{3}\right) \delta 207.2,137.8,130.08,128.3,127.9,126.4$, $125.9,101.9,99.6,32.7,31.8,22.3,17.1,15.5,14.0 ;{ }^{13} \mathrm{C}$ NMR (126 $\left.\mathrm{MHz}, \mathrm{C}_{6} \mathrm{D}_{6}\right) \delta 207.7,138.2,130.2,128.7,128.6,126.9,126.4,102.4$, 100.4, 33.1, 32.1, 22.7, 17.3, 15.7, 14.2; FTIR $\left(v_{\max } / \mathrm{cm}^{-1}\right) 3027$, 2956, 2925, 2863, 1933, 1492, 1444, 1367, 1027, 963, 758, 692, 604, 596; MS (EI, $m / z) 226$ (38, M+ ), 211 (21), 197 (9), 183 (100), 168 (52), 155 (83), 141 (55), 128 (40), 115 (37), 105 (28), 91 (33), 77 (21); HRMS (ESI) calcd for $\mathrm{C}_{17} \mathrm{H}_{23}\left(\mathrm{MH}^{+}\right)$227.1794, found 227.1795 .

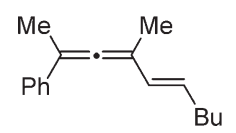

3ga. ${ }^{1} \mathrm{H}$ NMR (400 MHz, $\left.\mathrm{C}_{6} \mathrm{D}_{6}\right) \delta 7.32-7.50(\mathrm{~m}, 2 \mathrm{H})$, $7.10-7.20(\mathrm{~m}, 2 \mathrm{H}), 7.00-7.08(\mathrm{~m}, 1 \mathrm{H}), 6.20(\mathrm{dd}, J=15.8,1.4$ $\mathrm{Hz}, 1 \mathrm{H}), 5.59(\mathrm{dd}, J=15.6,7.0 \mathrm{~Hz}, 1 \mathrm{H}), 2.21-2.32(\mathrm{~m}, 1 \mathrm{H}), 2.03(\mathrm{~s}$, $3 \mathrm{H}), 1.89(\mathrm{~s}, 3 \mathrm{H}), 0.956(\mathrm{~d}, J=7.2 \mathrm{~Hz}, 3 \mathrm{H}), 0.953(\mathrm{~d}, J=7.2 \mathrm{~Hz}$, $3 \mathrm{H}) ;{ }^{13} \mathrm{C}$ NMR $\left(101 \mathrm{MHz}, \mathrm{C}_{6} \mathrm{D}_{6}\right) \delta 207.9,138.2$, 137.0, 128.7, $126.9,126.4,125.9,102.3,100.4,31.9,22.7,17.3,15.7$; FTIR ( $\nu_{\max }$ ) $\left.\mathrm{cm}^{-1}\right) 3027,2958,2867,1933,1597,1492,1465,1444,1366,1066$, 1029, 964, 758, 692, 603; MS (EI, $m / z) 212\left(47, \mathrm{M}^{+}\right), 197$ (100), 182 (32), 169 (31), 165 (29), 155 (48), 141 (36), 128 (35), 115 (29), 91 (24), 77 (15); HRMS (EI) calcd for $\mathrm{C}_{16} \mathrm{H}_{20}\left(\mathrm{M}^{+}\right)$212.1560, found 212.1551.

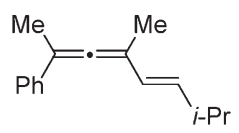

3ha. ${ }^{1} \mathrm{H}$ NMR (500 MHz, $\left.\mathrm{C}_{6} \mathrm{D}_{6}\right) \delta$ 7.46-7.50 (m, 4H), $7.14-7.23(\mathrm{~m}, 4 \mathrm{H}), 7.05-7.10(\mathrm{~m}, 2 \mathrm{H}), 6.16(\mathrm{dq}, J=15.5,1.5$ $\mathrm{Hz}, 1 \mathrm{H}), 5.50(\mathrm{dq}, J=15.8,6.5 \mathrm{~Hz}, 1 \mathrm{H}), 1.84(\mathrm{~s}, 3 \mathrm{H}), 1.61(\mathrm{dd}, J=$ 7.0, $1.5 \mathrm{~Hz}, 3 \mathrm{H}) ;{ }^{13} \mathrm{C} \mathrm{NMR}\left(126 \mathrm{MHz}, \mathrm{C}_{6} \mathrm{D}_{6}\right) \delta 209.5,137.9,129.2$, $129.1,128.8,127.4,125.4,110.2,103.5,18.4,15.6$; FTIR $\left(v_{\max }\right)$ $\mathrm{cm}^{-1}$ ) 3029, 2925, 1715, 1598, 1492, 1445, 962, 900, 839, 768, 738, 695, 630; MS (EI, $m / z) 246\left(100, \mathrm{M}^{+}\right), 231(24), 215(70)$, 202 (27), 189 (12), 165 (17), 153 (21), 128 (10), 115 (14), 91 (9), 77 (8); HRMS (EI) calcd for $\mathrm{C}_{19} \mathrm{H}_{18}\left(\mathrm{M}^{+}\right)$246.14030, found 246.13960 . 


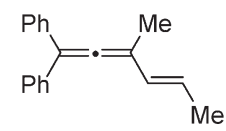

3ia. ${ }^{1} \mathrm{H}$ NMR (400 MHz, $\left.\mathrm{C}_{6} \mathrm{D}_{6}\right) \delta 7.30-7.35$ (m, 2H), 7.11$7.17(\mathrm{~m}, 2 \mathrm{H}), 7.04-7.10(\mathrm{~m}, 1 \mathrm{H}), 6.22(\mathrm{dq}, J=15.6,1.6 \mathrm{~Hz}, 1 \mathrm{H}), 5.42$ $(\mathrm{dq}, J=6.8,15.6 \mathrm{~Hz}, 1 \mathrm{H}), 1.79(\mathrm{~s}, 3 \mathrm{H}), 1.64(\mathrm{dd}, J=6.4,1.6 \mathrm{~Hz}, 3 \mathrm{H})$, 1.20 (s, 9H); ${ }^{13} \mathrm{C} \mathrm{NMR}\left(101 \mathrm{MHz}, \mathrm{C}_{6} \mathrm{D}_{6}\right) \delta 204.7,138.6,130.8,129.9$, 128.2 , 126.8, 123.6, 115.6, 101.0, 35.4, 30.3, 18.4, 16.2; FTIR $\left(v_{\max } / \mathrm{cm}^{-1}\right) 3018,2965,2902,2866,1442,1361,960,755,737,698$, 570; MS (EI, $m / z) 226\left(7, \mathrm{M}^{+}\right), 196(6), 181$ (6), 169 (100), 154 (72), 141 (25), 128 (24), 115 (19), 91 (26), 77 (12); HRMS (EI) calcd for $\mathrm{C}_{17} \mathrm{H}_{22}\left(\mathrm{M}^{+}\right)$226.1716, found 226.1710 .

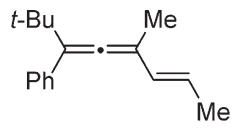

3ja. ${ }^{1} \mathrm{H}$ NMR (400 MHz, $\left.\mathrm{C}_{6} \mathrm{D}_{6}\right) \delta 7.24-7.28$ (m, 2H), 7.08$7.15(\mathrm{~m}, 2 \mathrm{H}), 6.90-7.04(\mathrm{~m}, 1 \mathrm{H}), 6.23(\mathrm{~s}, 1 \mathrm{H}), 6.12(\mathrm{~d}, J=15.6 \mathrm{~Hz}$, $1 \mathrm{H}), 5.50(\mathrm{dq}, J=15.6,6.4 \mathrm{~Hz}, 1 \mathrm{H}), 1.82(\mathrm{~d}, J=2.8 \mathrm{~Hz}, 3 \mathrm{H}), 1.60(\mathrm{~d}, J=$ $6.4 \mathrm{~Hz}, 3 \mathrm{H}) ;{ }^{13} \mathrm{C}$ NMR $\left(101 \mathrm{MHz}, \mathrm{C}_{6} \mathrm{D}_{6}\right) \delta 208.7,135.6,129.1,128.9$, 127.3, 127.1, 125.2, 104.2, 99.7, 18.3, 15.5; FTIR $\left(\nu_{\max } / \mathrm{cm}^{-1}\right) 3024$, 2871, 1932, 1598, 1495, 1448, 960, 825, 742, 692, 631; MS (EI, $m / z) 170\left(75, \mathrm{M}^{+}\right), 155$ (100), 141 (24), 128 (45), 115 (36), 102 (8), 91 (28), 77 (20); HRMS (EI) calcd for $\mathrm{C}_{13} \mathrm{H}_{14}\left(\mathrm{M}^{+}\right), 170.1090$, found 170.1084 .

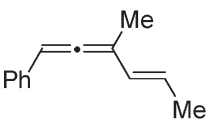

3la. ${ }^{1} \mathrm{H}$ NMR $\left(400 \mathrm{MHz}, \mathrm{CDCl}_{3}\right) \delta 7.42-7.36(\mathrm{~m}, 2 \mathrm{H}), 7.35-7.28$ $(\mathrm{m}, 2 \mathrm{H}), 7.22-7.16(\mathrm{~m}, 1 \mathrm{H}), 6.15(\mathrm{dt}, J=10.0,2.8 \mathrm{~Hz}, 1 \mathrm{H}), 5.92(\mathrm{ddq}$, $\left.J_{\mathrm{AB}}=15.2,10.4,1.2 \mathrm{~Hz}, 1 \mathrm{H}\right), 5.74\left(\mathrm{dq}, J_{\mathrm{AB}}=15.2,6.8 \mathrm{~Hz}, 1 \mathrm{H}\right)$, $2.49-2.41(\mathrm{~m}, 2 \mathrm{H}), 1.77(\mathrm{dd}, J=6.0,1.2 \mathrm{~Hz}, 2 \mathrm{H}),{ }^{13} \mathrm{C} \mathrm{NMR}(100 \mathrm{MHz}$, $\left.\mathrm{CDCl}_{3}\right) \delta 207.0,137.0,128.5,127.9,126.8,126.7,126.3,107.1,97.3$, 30.2, 30.0, 22.6, 18.4, 14.1; FTIR $\left(v_{\max } / \mathrm{cm}^{-1}\right) 2956,2926,2857,1931$, 1492, 1447, 1026, 962, 758, 692; MS (EI, $m / z) 212\left(5, \mathrm{M}^{+}\right), 170$ (48), 155 (100), 141 (28), 128 (19), 115 (20), 91 (21), 77 (6); HRMS (EI) calcd for $\mathrm{C}_{16} \mathrm{H}_{20}\left(\mathrm{M}^{+}\right)$212.1560, found 212.1554.

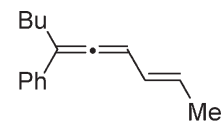

3ma. ${ }^{1} \mathrm{H}$ NMR (400 MHz, $\left.\mathrm{C}_{6} \mathrm{D}_{6}\right) \delta 7.39-7.45(\mathrm{~m}, 2 \mathrm{H})$, $7.15-7.21(\mathrm{~m}, 2 \mathrm{H}), 7.02-7.09(\mathrm{~m}, 1 \mathrm{H}), 6.51(\mathrm{dd}, J=17.2,10.4$ $\mathrm{Hz}, 1 \mathrm{H}), 5.12(\mathrm{dd}, J=17.2,1.2 \mathrm{~Hz}, 1 \mathrm{H}), 4.99(\mathrm{dd}, J=10.8,0.8 \mathrm{~Hz}$, $1 \mathrm{H}), 2.39(\mathrm{t}, J=7.6 \mathrm{~Hz}, 2 \mathrm{H}), 1.84(\mathrm{~s}, 3 \mathrm{H}), 1.51$ (quint, $J=7.6 \mathrm{~Hz}$, $2 \mathrm{H}), 1.33$ (sext, $J=7.6 \mathrm{~Hz}, 2 \mathrm{H}), 0.86(\mathrm{t}, J=7.6 \mathrm{~Hz}, 3 \mathrm{H}) ;{ }^{13} \mathrm{C} \mathrm{NMR}$ $\left(101 \mathrm{MHz}, \mathrm{C}_{6} \mathrm{D}_{6}\right) \delta 208.3,137.5,136.1,128.8,127.0,126.7,112.9$, 106.0, 103.9, 30.4, 30.3, 22.8, 14.9, 14.2; FTIR $\left(v_{\max } / \mathrm{cm}^{-1}\right) 3026$, 2957, 2929, 2859, 1931, 1613, 1493, 1455, 896, 759, 739, 694; MS (EI, $\left.m / z, \mathrm{M}^{+}\right): 212\left(2, \mathrm{M}^{+}\right), 197$ (1), 170 (54), 155 (100), 141 (35), 128 (23), 115 (31), 91 (23), 77 (12); HRMS (EI) calcd for $\mathrm{C}_{16} \mathrm{H}_{20}$ $\left(\mathrm{M}^{+}\right)$212.1560, found 212.1560.

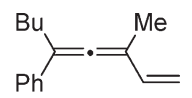

\section{ASSOCIATED CONTENT}

S Supporting Information. Calculated DFT parameters and ${ }^{1} \mathrm{H}$ and ${ }^{13} \mathrm{C}$ spectra of all products. This material is available free of charge via the Internet at http://pubs.acs.org.

\section{AUTHOR INFORMATION}

\section{Corresponding Author}

*E-mail: leventartok@iyte.edu.tr.

\section{ACKNOWLEDGMENT}

The financial support of The Scientific and Technological Research Council of Turkey and Federal Ministry of Education and Research (Germany) via the Intensified Cooperation Program (210T092), a Fellowship Program by Deutscher Akademischer Austauschdienst, and a Scientific Exchange Fellowship Program by The Scientific and Technological Research Council of Turkey and Deutsche Forschungs Gemeinschaft to L.A. are gratefully acknowledged. We thank Ms. I. Özçelik, Mr. S. Günnaz, and Dr. W. Hiller for NMR analyses, Dr. H. Özgener for FTIR analyses, and Dr. R. Eanes for proofreading.

\section{REFERENCES}

(1) (a) Miyaura, N.; Suzuki, A. Chem. Rev. 1995, 95, 2457. (b) Suzuki, A. J. Organomet. Chem. 1999, 576, 147.(c) Suzuki, A. Metalcatalyzed Cross-Coupling Reactions; Diederich, F., Stang, P. J., Eds.; Wiley-VCH: Weinheim, 1998; p 49.

(2) (a) Brown, H. C. Organic Syntheses via Boranes; Wiley-Interscience: New York, 1975. (b) Matteson, D. S. Stereodirected Synthesis with Organoboranes; Springer-Verlag: Berlin, 1995.

(3) Boronic Acids; Hall, D. G., Ed.; Wiley-VCH: Weinheim, 2005.

(4) Reviews: (a) Fagnou, K.; Lautens, M. Chem. Rev. 2003, 103, 169. (b) Hayashi, T.; Yamasaki, K. Chem. Rev. 2003, 103, 2829.

(5) (a) Sakai, M.; Hayashi, H.; Miyaura, N. Organometallics 1997, 16, 4229. (b) Takaya, Y.; Ogasawara, M.; Hayashi, T.; Sakai, M.; Miyaura, N. J. Am. Chem. Soc. 1998, 120, 5579. (c) Sakuma, S.; Miyaura, N. J. Org. Chem. 2001, 66, 8944. (d) Sakuma, S.; Sakai, M.; Itooka, R.; Miyaura, N. J. Org. Chem. 2000, 65, 5951. (e) Itooka, R.; Iguchi, Y.; Miyaura, N. J. Org. Chem. 2003, 68, 6000. (e) Takaya, Y.; Senda, T.; Kurushima, H.; Ogasawara, M.; Hayashi, T. Tetrahedron: Asymmetry 1999, 10, 4047.(f) Miyaura, N. In Organoboranes in Syntheses; Ramachandran, P. V., Brown, H. C., Eds.; ACS Symposium Series 783; American Chemical Society: Washington, DC, 2001; Chapter 7, pp 94-107. (g) Hayashi, T. Synlett 2001, 879. (h) Bolm, C.; Hildebrand, J. P.; Munñiz, K.; Hermanns, N. Angew. Chem., Int. Ed. 2001, 40, 3284. (i) Hayashi, T.; Takahashi, M.; Takaya, Y.; Ogasawara., M. J. Am. Chem. Soc. 2002, 124, 5052. (j) Hayashi, T. Bull. Chem. Soc. Jpn. 2004, 77, 13. (k) Christoffers, J.; Koripelly, G.; Rosiak, A.; Rössle, M. Synthesis 2007, 1279.

(6) Sakai, M.; Ueda, M.; Miyaura, N. Angew. Chem., Int. Ed. Engl. 1998, 37, 3279.

(7) (a) Hayashi, T.; Inoue, K.; Taniguchi, N.; Ogasawara, M. J. Am. Chem. Soc. 2001, 123, 9918. (b) Lautens, M.; Yoshida, M. Org. Lett. 2002, 4, 123.

(8) (a) Murakami, M.; Igawa, H. Helv. Chim. Acta 2002, 85, 4182. (b) Miura, T.; Shimada, M.; Ku, S.-Y.; Tamai, T.; Murakami, M. Angew. Chem., Int. Ed. 2007, 46, 7101. (c) Miura, T.; Shimada, M.; de Mendoza, P.; Deutsch, C.; Krause, N.; Murakami, M. J. Org. Chem. 2009, 74, 6050. (d) Yoshida, M.; Hayashi, M.; Matsuda, K.; Shishido, K. Heterocycles 2009, 77, 193.

(9) Lautens, M.; Dockendorff, C.; Fagnou, K.; Malicki, A. Org. Lett. 2002, 4, 1311.

(10) (a) Goré, J.; Dulcere, J. P. J. Chem. Soc., Chem. Commun. 1972, 866. (b) Dulcere, J. P.; Goré, J.; Roumestant, M. L. Bull. Soc. Chim. Fr. 1974, 1119. (c) Bertrand, M.; Dulcere, J. P.; Gil, G.; Roumestant, M. L. Tetrahedron Lett. 1979, 20, 1845. (d) Dulcere, J. P.; Grimaldi, J.; Santelli, M. Tetrahedron Lett. 1981, 22, 3179.

(11) (a) Purpura, M.; Krause, N. Eur. J. Org. Chem. 1999, 267. (b) Krause, N.; Purpura, M. Angew. Chem., Int. Ed. 2000, 39, 4355.

(12) Recently, a rhodium-catalyzed 1,6-addition of arylboronic acids to enynamides leading to axially chiral allenylsilanes was described: Nishimura, T.; Makino, H.; Nagaosa, M.; Hayashi, T. J. Am. Chem. Soc. 2010, 132, 12865 . 
(13) Akpınar, G. E.; Kus, M.; Uçüncü, M.; Karakuș, E.; Artok, L. Org. Lett. 2011, 13, 748.

(14) (a) Moriya, T.; Miyaura, N.; Suzuki, A. Synlett 1994, 149. (b) Yoshida, M.; Ueda, H.; Ihara, M. Tetrahedron Lett. 2005, 46, 6705.

(15) A number of bases, such as $\mathrm{NEt}_{3}, \mathrm{NaOAc}, \mathrm{K}_{2} \mathrm{CO}_{3}, \mathrm{NaOH}$, $\mathrm{K}_{3} \mathrm{PO}_{4}$, and $\mathrm{NaHCO}_{3}$, were also tested. However, the reactions resulted in incomplete conversion of the $(Z)$-enyne 1.1a and the formation of byproducts.

(16) No successful results could be obtained by the use of 1-naphthyl-, 3-pyridyl-, and 1-pentenylboronic acids.

(17) The reactions of enynes with tert-butyl, $\mathrm{SiMe}_{3}$, or $\mathrm{Ph}$ substituents on the $\mathrm{R}^{1}$ position $\left(\mathrm{R}^{2}=\mathrm{R}^{3}=\mathrm{Me}\right)$ and of a primary enyne acetate $\left(\mathrm{R}^{1}=\mathrm{R}^{2}=\mathrm{Me}, \mathrm{R}^{3}=\mathrm{H}\right)$ were highly sluggish and afforded the related vinylallene products in very low yields, usually $<20 \%$. The reactions of enynes with $R^{1}=H, R^{2}=R^{3}=M e$ and $R^{1}=R^{3}=M e, R^{2}=H$ gave complex mixtures.

(18) The Lowe-Brewster rule was used to correlate the sign of optical rotation with the absolute configuration of the allene: (a) Lowe, G. Chem. Commun. 1965, 411. (b) Brewster, J. H. Top. Stereochem. 1967, $2,33$.

(19) (a) Ogasawara, M.; Fan, L.; Ge, Y.; Takahashi, T. Org. Lett. 2006, 8, 5409. (b) Ogasawara, M.; Okada, A.; Watanabe, S.; Fan, L.; Uetake, K.; Nakajima, K.; Takahashi, T. Organometallics 2007, 26, 5025.

(20) An example of $\delta$-elimination of Rh and an oxygen functionality was previously reported:Miura, T.; Shimizu, H.; Igarashi, T.; Murakami, M. Org. Biomol. Chem. 2010, 8, 4074.

(21) In order to ascertain whether the product $3 \mathbf{j a}$ was formed by desilylation of the expected primary silyl-substituted vinylallene product or arylative substitution of the desilylated form $(\mathbf{1 . 2 j})$ of silyl substituted

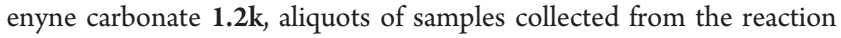
progress of $1.2 \mathrm{k}$ and phenylboronic acid were subjected to GC-MS analyses. The only determined structures were the initial enyne substrate $1.2 \mathrm{k}$ and the product $3 \mathrm{ja}$. This may indicate that the desilylation process occurred primarily from the intermediates of the reaction cycle.

(22) Granberg, K. L.; Bäckvall, J.-E. J. Am. Chem. Soc. 1992, 114,6858 .

(23) Chloroform solutions of the enyne substrates and the vinylallene products are not so stable and undergo gradual decomposition. Nevertheless, they are more stable in benzene.

(24) Ukai, T.; Kawazura, H.; Ishii, Y.; Bonnet, J. J.; Ibers, J. A. J. Organomet. Chem. 1974, 65, 253.

(25) (a) Giordano, G.; Crabtree, R. H. Inorg. Synth. 1979, 19, 218. (b) Artok, L.; Kus, M.; Ürer, B. N.; Türkmen, G.; Aksın-Artok, Ö. Org. Biomol. Chem. 2010, 8, 2060.

(26) Uson, R.; Oro, L. A.; Cabeza, J. A. Inorg. Synth. 1985, 23, 126.

(27) Babudri, F.; Fiandanese, V.; Marchese, G.; Punzi, A. Tetrahedron 2000, 56, 327.

(28) Becke, A. D. J. Chem. Phys. 1993, 98, 5648.

(29) Frisch, M. J. et al. Gaussian 09, revision A.02; Gaussian, Inc.: Wallingford, CT, 2009. 